\title{
Chapter 5 \\ Efficacy of Trans-geographic \\ Observational Network Design \\ for Revelation of Growth Pattern \\ in Mountain Forests Across Europe
}

\author{
H. Pretzsch, T. Hilmers, E. Uhl, M. del Río, A. Avdagić, K. Bielak, A. Bončina, \\ L. Coll, F. Giammarchi, K. Stimm, G. Tonon, M. Höhn, M. Kašanin-Grubin, \\ and R. Tognetti
}

\begin{abstract}
Understanding tree and stand growth dynamics in the frame of climate change calls for large-scale analyses. For analysing growth patterns in mountain forests across Europe, the CLIMO consortium compiled a network of observational plots across European mountain regions. Here, we describe the design and efficacy of this network of plots in monospecific European beech and mixed-species stands of Norway spruce, European beech, and silver fir.
\end{abstract}

\footnotetext{
H. Pretzsch $(\triangle) \cdot T$. Hilmers

Chair of Forest Growth and Yield Science, Department of Life Science Systems, TUM School of Life Sciences, Technical University of Munich, Freising, Germany

e-mail: hans.pretzsch@tum.de; torben.hilmers@tum.de

E. Uhl · K. Stimm

Chair of Forest Growth and Yield Science, Department of Life Science Systems, TUM School of Life Sciences, Technical University of Munich, Freising, Germany
}

Bavarian State Institute of Forestry (LWF), Freising, Germany

e-mail: enno.uhl@tum.de; kilian.stimm@tum.de; kilian.stimm@1wf.bayern.de

M. del Río

INIA, Forest Research Centre, Madrid, Spain

iuFOR, Sustainable Forest Management Research Institute, University of Valladolid \& INIA, Valladolid, Spain

e-mail: delrio@inia.es

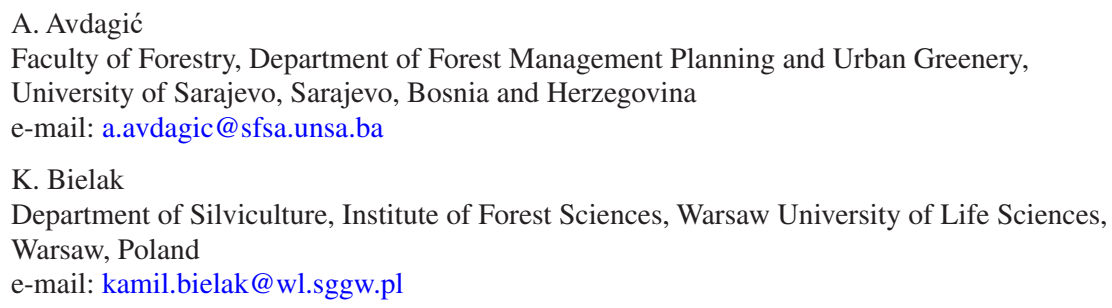


First, we sketch the state of the art of existing monitoring and observational approaches for assessing the growth of mountain forests. Second, we introduce the design, measurement protocols, as well as site and stand characteristics, and we stress the innovation of the newly compiled network. Third, we give an overview of the growth and yield data at stand and tree level, sketch the growth characteristics along elevation gradients, and introduce the methods of statistical evaluation. Fourth, we report additional measurements of soil, genetic resources, and climate smartness indicators and criteria, which were available for statistical evaluation and testing hypotheses. Fifth, we present the ESFONET (European Smart Forest Network) approach of data and knowledge dissemination. The discussion is focussed on the novelty and relevance of the database, its potential for monitoring, understanding and management of mountain forests toward climate smartness, and the requirements for future assessments and inventories.

In this chapter, we describe the design and efficacy of this network of plots in monospecific European beech and mixed-species stands of Norway spruce, European beech, and silver fir. We present how to acquire and evaluate data from individual trees and the whole stand to quantify and understand the growth of mountain forests in Europe under climate change. It will provide concepts, models, and practical hints for analogous trans-geographic projects that may be based on the existing and newly recorded data on forests.

\author{
A. Bončina \\ Biotechnical Faculty, Department of Forestry and Renewable Forest Resources, \\ University of Ljubljana, Ljubljana, Slovenia \\ e-mail: andrej.boncina@bf.uni-lj.si \\ L. Coll \\ Department of Agriculture and Forest Engineering (EAGROF), University of Lleida, \\ Lleida, Spain \\ Joint Research Unit CTFC-AGROTECNIO, Solsona, Spain \\ e-mail: 1luis.coll@udl.cat \\ F. Giammarchi · G. Tonon \\ Faculty of Science and Technology, Free University of Bolzano-Bozen, Bolzano, Italy \\ e-mail: francesco.giammarchi@ unibz.it; giustino.tonon@unibz.it \\ M. Höhn \\ Faculty of Horticultural Science, Department of Botany, SZIU, Budapest, Hungary \\ e-mail: hohn.maria@kertk.szie.hu \\ M. Kašanin-Grubin \\ Institute for Chemistry, Technology and Metallurgy, University of Belgrade, Belgrade, \\ Serbia \\ e-mail: mkasaningrubin@chem.bg.ac.rs \\ R. Tognetti \\ Dipartimento di Agricoltura, Ambiente e Alimenti, Università degli Studi del Molise, \\ Campobasso, Italy \\ e-mail: tognetti@unimol.it
}


tree growth

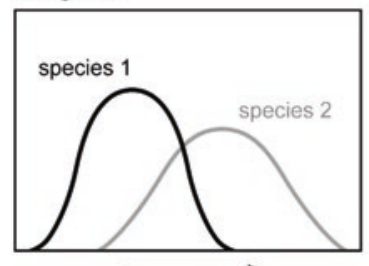

(a)

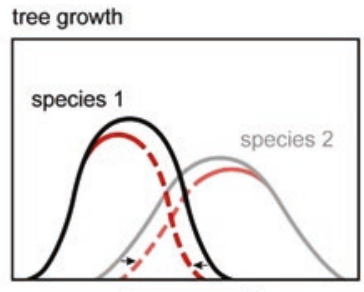

environmental conditions

(b)

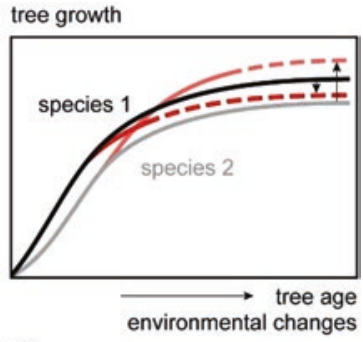

(c)

Fig. 5.1 The effect of changing environmental conditions on species growth. (a) Environmental changes may be detrimental for species 1 but advantageous for species 2 due to better match of fundamental niche. (b) The fundamental niches are modified by interspecific synecological effects. (c) The course of growth of trees and species-specific ranking may be modified within the lifetime of trees due to environmental changes

\subsection{Assessing the Climate Sensitivity of the Growth of European Mountain Forests}

Environmental changes (indicated by an arrow in Fig. 5.1a) may promote a species with a more suitable fundamental niche (species 2) and reduce the growth of species 1 (Fig. 5.1a). Tree growth indicates the effects of climate and other environmental conditions on the production, adaptation, and mitigation of forest stands. In Figure 5.1, we indicate the fundamental niche by a monocausal gradient along the abscissa and the growth as indicator for fitness on the ordinate.

In mixed stands, the species also encounter interspecific competition with additional synecological disadvantages, as indicated by a narrowing of the fundamental to the real niche in Figure 5.1b. Due to their longevity, trees may be exposed to environmental changes over centuries and modify the course of their growth, as well as species-specific competition and facilitation (Fig. 5.1c).

For trees in the northern latitudes or in the higher elevations of mountain areas, this means that they change their growth due to the modified potential growing conditions, and in addition, they may face the new competition effects by other species of the ecosystem. Repeated observations in permanent plots are, therefore, necessary to confirm or confute the status of monitored trees and their growth trends over time (Franklin 1989). These plots may be also useful for re-examining ecological theories (e.g. disturbance ecology, forest succession) and temporal series (e.g. biomass accumulation, tree mortality) in the framework of environmental changes (van Mantgem and Stephenson 2007; Harmon and Pabst 2015).

In mountain forests, at the edge of their ecological amplitude, little changes of environmental conditions may trigger strong non-linear effects on tree growth superimpose by additional competition effects due to strengthening of neighbours, which grow in the proximity and are better adapted to the new conditions (Pretzsch et al. 2020a, b). 
This project deals with the effects of climate changes on the growth dynamics of mountain forest ecosystems that are so far much less understood than the ones in northern latitudes, although they may undergo even worse changes regarding the stability and ecosystem service provision (Tognetti et al. 2017). In addition to the non-linear reaction pattern at the left or right branch of the ecological niche, mountain forests are very susceptible to climate changes due to their harsh site conditions, slopes, mechanical unstable conditions, remoteness, and thus limiting accessibility to mitigating silvicultural measures.

The concept and data acquisition should provide the basis for answering the following questions and scrutinizing the following hypotheses:

(i) How is the state of the productivity, vitality, and climate smartness of mountain forests in Europe?

(ii) How did the stand productivity of mountain forests change in the recent centuries according to records from long-term experiments and information extracted from increment core analyses?

(iii) How did the growth of the main tree species change in the recent centuries and were any changes of tree growth depending on the elevation above sea level?

This chapter presents the interdisciplinary database and trans-geographic plot network, underlying recent research articles (Hilmers et al. 2019; Pretzsch et al. 2020a, b; Torresan et al. 2020; del Río et al. 2021).

\subsection{State of the Art of Monitoring and Observational Approaches}

The analysis of forest growth is one of the fundamentals of forestry and forest science, so there are different well-established approaches for obtaining the required data from forest stands (e.g. Kangas and Maltamo 2006; Pretzsch 2009; Ferretti and Fischer 2013). Here, we briefly introduce the main concepts and approaches, underlining the most relevant aspects of data sources, to properly analyse the growth trends and responses to disturbances and extreme weather events in mountain forests under global climate change. We address different organization levels across both temporal and spatial scales, and we present selected methodological approaches.

Organizational Level The most common approach used for analysing long-term growth trends is the tree level, since tree coring allows easily obtaining long treering series. However, it is not possible to evaluate the climate change impacts on forest dynamics without addressing the growth at stand level, which implicitly considers ingrowth and mortality. When stand-level data are not available, tree-level data covering stand size distribution can be an acceptable compromise, since growth response of trees to climate, disturbance, and extreme events varies significantly among social classes of trees (Pretzsch et al. 2018). Given that biomass accumulation in forests depends on the balance between growth (carbon sequestration) and 
mortality (carbon loss) of trees, monitoring changes at an individual tree level may enable a better understanding of forest-climate feedback and post-disturbance dynamics (see Chap. 10 of this book: Tognetti et al. 2021). Studies at lower levels, such as organ or cell growths, may provide additional information to better understand forest growth variation with climate change, but in general, they are not feasible for large samples. On the other hand, applications for forest management and biodiversity conservation may require detailed data representing large spatial extents, which can be obtained through remote sensing (see Chap. 11 of this book: Torresan et al. 2021).

Spatial Scale Forest growth data can be gathered from local to global scale. Local or regional growth trends analysis can be very relevant for their use at these scales and especially when the study area represents the rear edge of the species distribution (e.g. Dorado-Liñán et al. 2020; Hernández et al. 2019). However, transgeographic networks across large areas are needed to identify general patterns and main drivers of growth trends in regard to gradual and episodic environmental stress (Gazol and Camarero 2016).

Temporal Scale Two aspects related to temporal scale must be considered, the temporal resolution and the continuity, i.e. temporal vs. permanent plots. Regarding the former, daily and intra-annual tree growth may reveal useful information about climate drivers and growth, but for analysing growth trends and forest dynamics, annual resolution is generally the most robust option. Lower resolution such as 5- or 10 -year periods may not always well describe the growth patterns; however, longterm series can also provide unique information on forest growth trends (Pretzsch et al. 2014; Hilmers et al. 2019). Nevertheless, temporal resolution is often linked to continuity, since long-term series at stand level inevitably involves permanent plots. Advantages and disadvantages of temporal and permanent plots have been frequently discussed (e.g. Gadow 1999), but unquestionably, for exploring forest growth trends and understanding climate change consequences, long-term experimental plots, where stand history has been recorded, offer invaluable information (Pretzsch et al. 2019). Therefore, in this respect, long-term experiments so far outperform the information potential of inventory plots that have been increasingly established during the last two decades under the umbrella of National Forest Inventories in Europe.

MethodologicalApproach According to the used concept, experimental approaches can be classified as observational or manipulated ones. Traditionally, manipulated experiments with a statistical design and control of factors have been accepted as the correct way to identify the causal effects. However, the increasing capacity of obtaining large amounts of data strengthens the ability of observational approaches for testing hypotheses, so they currently are an essential source to analyse global environmental problems at a large spatial-temporal scale (Sagarin and Pauchard 2010). In forests, the most critical part of observational data is that often the stand management history is unknown, although this issue can be overcome by long-term monitoring. Observational approaches have been classified as inventory-based or 
exploratory methods (Bauhus et al. 2017, pp. 53-64). Inventoried-based approaches follow different sampling designs, generally systematic sampling, with the aim to gain in representativeness of the whole studied population. On the contrary, exploratory approaches distribute samples along gradients of specific factors to study their causal relationship. For our aim, spatial distribution of samples/plots may be designed to cover different site conditions, as tree and stand growth, as well as the impact of climate change, are strongly dependent on them. For this, transects along environmental gradients are particularly useful (Pretzsch et al. 2014). In mountain areas, altitudinal transects are generally the most efficient option (Ettinger et al. 2011).

Ideally, to study growth trends and responses to extreme events, data should cover all kinds of site conditions, across a large geographical area, during a long period of time, and focussing at least on tree and stand level, and at annual resolution, but of course this is not realistic. Often there are available data, which cover a large spatial scale but short temporal scale or vice versa, so integrating approaches are needed (Sagarin and Pauchard 2010), like the network presented in Section 5.3. In Section 5.7, further discussion of advantages and disadvantages of different approaches is included, with special emphasis on long-term experimental plots.

There are several examples of large forest growth databases based on different approaches. Ruiz-Benito et al. (2020) reviewed the available data sources in Europe for modelling climate change impacts on forests, including growth databases, such as the following: National Forest Inventories (Tomppo et al. 2010); the ICP forests European network (Ferretti and Fischer 2013); the DEIMS-SDR, including the Long-Term Research sites (LTER) (Wohner et al. 2019); the International TreeRing Data Bank (ITRDB) (Grissino-Mayer and Fritts 1997); networks of long-term experiments, like the Northern European Database of Long-Term Experiments (NOLTFOX) and the worldwide ForestGEO network (Anderson-Teixeira et al. 2015); and different remote sensing data sources.

Some of these datasets have their origin in institutional collaboration among countries, but the increasing number of initiatives for sharing research data, as the recent Global Forest Biodiversity Initiative (GFB), is remarkable (Liang et al. 2016). Although nowadays the publication of research data is often demanded by many funding institutions and publishing houses, these initiatives suppose a good opportunity for large-scale analysis, as they compile the information in a common platform.

\subsection{The CLIMO Design of Transnational Observational Network}

\subsubsection{Study Design and Data Used}

Tree species distribution and competitiveness in mountain forest ecosystems are strongly determined by geographic and topographic factors (Fig. 5.2). It is expected that climate change may affect the growth performance of tree species in mountain regions differently but possibly leading to a modification of the fitness and 


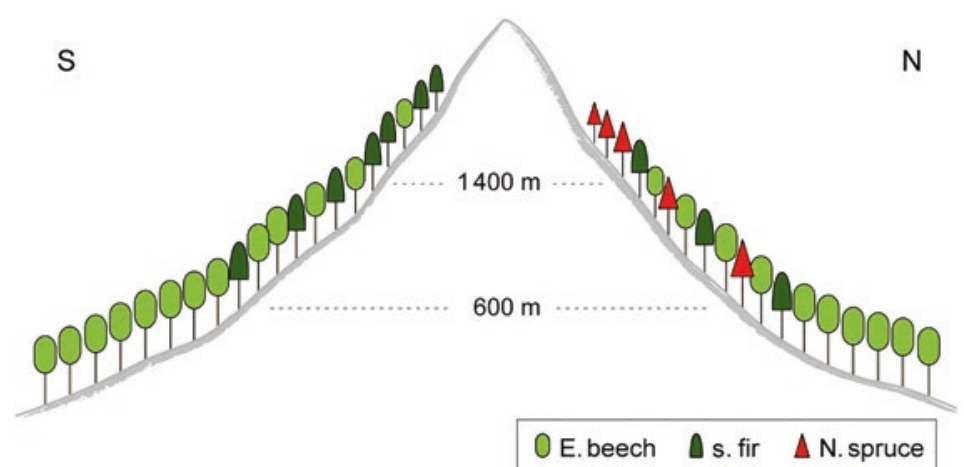

Fig. 5.2 Elevation and aspect are the main factors shaping species distribution and stand composition in mountain forests

subsequently to a change in tree species composition and distribution (Becker and Bugmann 2001). A comprehensive view about the general performance and a potentially recent change in performance of European mountain forests is widely missing. Two empirical studies were designed in the frame of CLIMO to improve the knowledge of historic and recent growth dynamics in mountain forests. We selected two most common types of mountain forests. In study 1 , we investigated mountain mixed forests, comprising Norway spruce, silver fir, and European beech, while in case of study 2, we focused on monospecific beech and beech-dominated mixed stands. Both studies were intended to analyse the growth and growth trends on stand and tree level. Short- and long-term growth trends were analysed against various factors concerning stand structure (e.g. tree species composition, density, diameter distribution) and site factors (e.g. climate, elevation).

Two different data sources were utilized to create a transnational observational network and to compile respective datasets for the analyses. Concerning mixed mountain forests, existing stand- and tree-level data from repeated inventoried longterm observational plots across European mountain regions were collected (Sect. 5.3.4). In contrast, the network of temporary plots representing geographic and elevation gradients in European beech-dominated stands were established and inventoried. Additionally, tree cores were obtained in both cases (study 1 and 2). Tree cores were used to analyse species-specific growth trends and growth reaction to drought events as well as to reconstruct recent stand-level performance on temporary plots applying the method described by Heym et al. (2018).

The analysis using temporary plots in beech-dominated stands followed two main research lines (RL). The first (RL1) focused on the effect of stand structural parameters on beech performance at tree and stand level. The second (RL2) intended to reveal the effect of elevation on growth rate and growth temporal trends. In the former case, two plots per site were established having similar elevation and growing conditions and differing in stand structural characteristics. In the latter case, two similarly structured stands at different elevations, but growing in similar conditions, were sampled. In some sites, both RLs were combined, i.e. two structures at each elevation. 
When designing trans-geographic studies, used local datasets should follow the common standards. In particular, when involving new (temporary) sample data, the common standards for site selection, data sampling, and data analysis are a prerequisite to facilitate analyses and to reduce post-processing effort. Following the keystones of common standards guarantees (i) the strengthening of statistical analysis options by enhancing the parameter specific number of degrees of freedom, (ii) the comparability of results with those from existing studies, (iii) confirmability of the analyses, and (iv) the usability of the data for follow-up studies.

\subsubsection{Site Selection Criteria}

Before plot establishment, in situ criteria for site and stand selection need to be defined. They have to be deduced from the study-specific research questions and hypotheses. These criteria have to delineate the subject of research and identify which factors to be included in the analyses are necessarily kept constant and which are allowed to vary. In study 2 , which utilized the newly established plots, site selection was limited to mountainous regions. However, stand selection per site was more in-depth, requiring a specific current stand age range for all plots. Concerning the RL1, the two plots of a single site were allowed only to differ in stand structural characteristics (e.g. density, species composition) whereas keeping site conditions and elevation constant. Concerning the RL2, two similar structured stands, having same topographic features, but only discriminated in elevation ( $\mathrm{min} .200 \mathrm{~m}$ ), had to be selected per site (Table 5.1, cf. Fig. 5.3).

\subsubsection{Plot Metadata}

After plot establishment, a precise and detailed description of the plot and topographic characteristics of plots, as well as environmental conditions, is necessary (Table 5.2). Coordinates and plot shape information guarantee the permanent identification of the single plot location. Topographic characteristics should be as detailed as possible and provide at least information about the factors needed for data analysis. The degree of detail concerning information on soil conditions and historic and current climatic characteristics is again dependent on the aim of the analyses.

\subsubsection{Tree Inventory and Dendrochronology}

The set of tree-specific variables to be collected per plot depends on the detail needed for the intended analyses. In CLIMO, empirical studies concerned productivity and structure on both tree and stand level. Thus, beside the standard stand data, also single-tree information is required to address and interlink both levels 
Table 5.1 Exemplary site selection criteria for temporal plots used in CLIMO study 2

\begin{tabular}{|c|c|c|c|}
\hline Category & Criteria & RL1 & RL2 \\
\hline \multirow[t]{3}{*}{ Geographic } & $\begin{array}{l}\text { Specification } \\
\text { of location }\end{array}$ & European mountain regions $\mathrm{s}^{\mathrm{a}}$ ) & $\begin{array}{l}\text { European mountain } \\
\text { regions }^{\text {a) }} \text { ) }\end{array}$ \\
\hline & Elevation & Equal for two plots per site & $\begin{array}{l}\text { Min. } 200 \mathrm{~m} \text { in difference } \\
\text { between two plots per site }\end{array}$ \\
\hline & $\begin{array}{l}\text { Site factors } \\
\text { (slope, aspect, } \\
\text { soil type) }\end{array}$ & Constant for min. Two plots per site & $\begin{array}{l}\text { Constant for min. Two plots } \\
\text { per site }\end{array}$ \\
\hline \multirow[t]{5}{*}{$\begin{array}{l}\text { Forest } \\
\text { stand }\end{array}$} & $\begin{array}{l}\text { Species } \\
\text { composition }\end{array}$ & $\begin{array}{l}\text { Monospecific beech stands - basal } \\
\text { area of beech } \geq 90 \% \\
\text { Beech-dominated mixed stands - } \\
\text { basal area of beech }>30 \% \text { and }<70 \%\end{array}$ & $\begin{array}{l}\text { Monospecific beech } \\
\text { stands - basal area of beech } \\
\geq 90 \%\end{array}$ \\
\hline & Stand age & $\begin{array}{l}\text { Similar for the dominant trees and } \\
\text { between } 70 \text { and } 100 \text { years }\end{array}$ & $\begin{array}{l}\text { Similar for the dominant } \\
\text { trees and between } 70 \text { and } \\
100 \text { years }\end{array}$ \\
\hline & $\begin{array}{l}\text { Management } \\
\text { history }\end{array}$ & Unmanaged for at least 15 years & $\begin{array}{l}\text { Unmanaged for at least } \\
15 \text { years }\end{array}$ \\
\hline & Plot size & Min. 0.1 ha, min. 50 trees per plot & $\begin{array}{l}\text { Min. } 0.1 \text { ha, } \min .50 \text { trees } \\
\text { per plot }\end{array}$ \\
\hline & $\begin{array}{l}\text { Stand } \\
\text { structure }\end{array}$ & Different between two plots per site & $\begin{array}{l}\text { Constant between two plots } \\
\text { per site }\end{array}$ \\
\hline
\end{tabular}

${ }^{\mathrm{a}}$ Mountain region followed the respective national definition - mountain definition can be constrained by a combination of elevation and ruggedness (Kapos et al. 2000) or by ruggedness of terrain only, irrespective of elevation (Körner et al. 2011)

(Table 5.3). Additional information can still be planned, once monitoring plots have been established, such as repeated observations of reproductive structures, phenological phases, physiological conditions, and mortality rates, to select novel indicators for assessing climate smartness of forests over time.

Temporary plots, measured for the first time, like in the case of study 2 , generally lack information about recent stand growth. However, stand-level increment can be reconstructed with the help of tree-ring chronologies derived from tree cores (Heym et al. 2018). As annual increment varies among trees of different social classes (del Rio et al. 2014; Torresan et al. 2020), it is important that the sampled trees cover the whole spectrum of the stand diameter distribution (Cherubini et al. 1998). To consider mortality when estimating stand productivity, dead trees have to be included in tree inventory, also estimating the probable year of death. In managed stands, an inventory of stumps and an estimate of the year of thinning improve the accuracy of reconstruction. However, selecting stands that have not been managed during the last 15 years may improve the stand growth reconstruction.

\subsection{Network, Locations, Site Characteristics}

In total, the trans-geographic network made it possible to collect and homogenize data from 159 observational plots in 14 countries across Europe (Fig. 5.3, Table 5.4). Plots are located mainly in fully stocked, un-thinned, or slightly thinned forest 


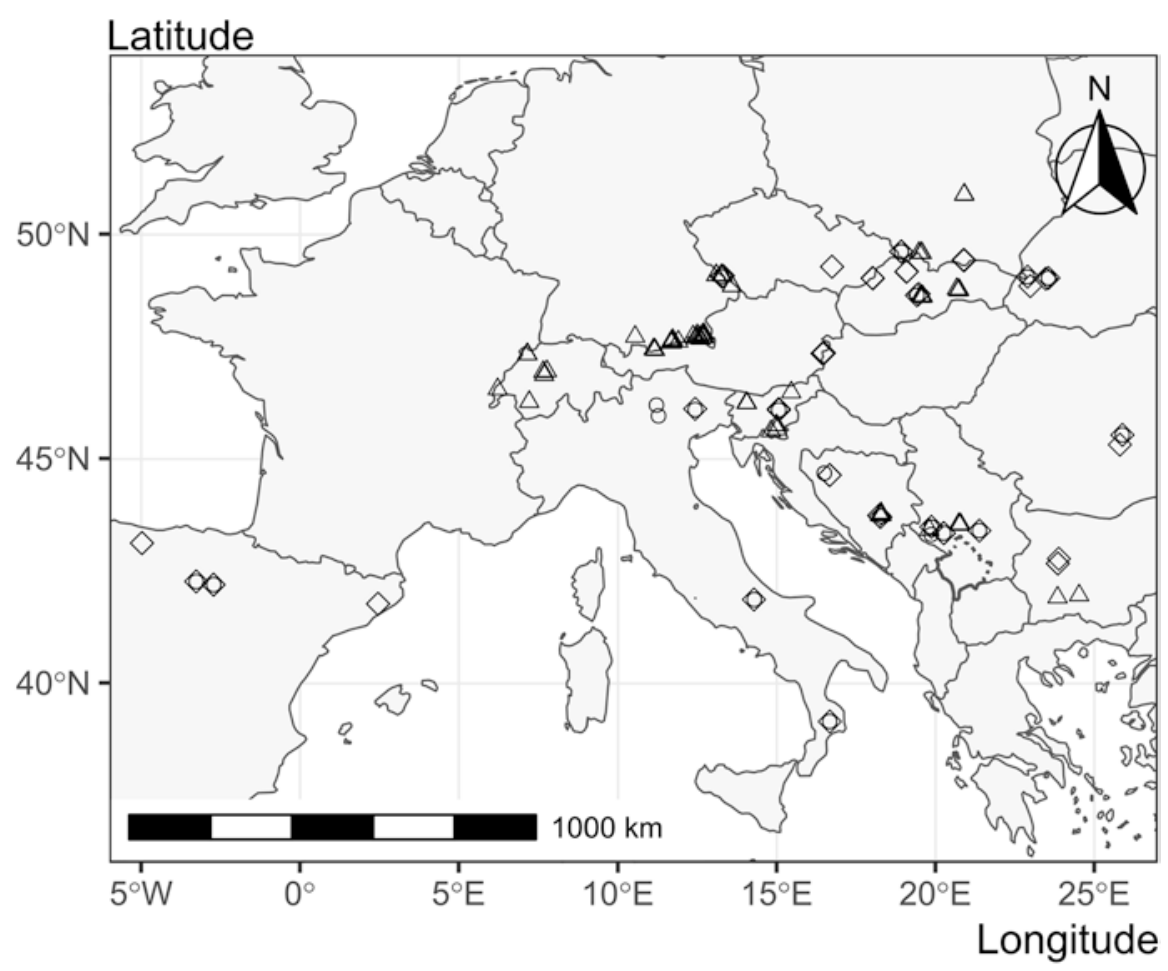

Fig. 5.3 Location of the 89 long-term observational plots in mixed mountain forests (triangles) and 72 temporary observational plots in monospecific stands of beech in mountain areas (rhombuses; $n=48$ ) and mixed mountain forests (circles; $n=22$ ) of 14 countries. The dataset covers mountain forests in Bosnia and Herzegovina, Bulgaria, Czech Republic, Germany, Hungary, Italy, Poland, Romania, Serbia, Slovakia, Slovenia, Spain, Switzerland, and Ukraine

stands that reflect natural dynamics and climatic variability. The dataset covered the mountain forests in Bosnia and Herzegovina, Bulgaria, Czech Republic, Germany, Hungary, Italy, Poland, Romania, Serbia, Slovakia, Slovenia, Spain, Switzerland, and Ukraine. The observational network comprises 89 long-term plots in mixed mountain forests mainly consisting of European beech (Fagus sylvatica L.), Norway spruce (Picea abies (L.) Karst), and silver fir (Abies alba Mill.), which have been under observation for at least 30 years. In addition, 70 temporary observational plots were established (see Sect. 5.3.3), representing 48 monospecific stands of European beech and 22 mixed mountain forests. In the latter case, European beech was mainly mixed with Norway spruce and silver fir, but studied plots included other admixed species as well, such as Scots pine (Pinus sylvestris L.), sycamore maple (Acer pseudoplatanus L.), European larch (Larix decidua Mill.), and European hornbeam (Carpinus betulus L.). Except for Scots pine and sycamore maple, these minor species, however, represent less than $10 \%$ of the stand basal area. All the study sites are located in mountain regions, from Picos de Europa (Spain) in the west to the Southern Carpathians (Romania) in the east, and from the Tatras (Poland) in the north to the Apennines (Italy) in the south. Elevations vary 
5 Efficacy of Trans-geographic Observational Network Design for Revelation...

Table 5.2 Exemplary list of single plot-specific information (metadata)

\begin{tabular}{|c|c|c|c|}
\hline Information & Criteria & & Unit \\
\hline \multirow[t]{5}{*}{ Location } & $\begin{array}{l}\text { Site } \\
\text { identification }\end{array}$ & Unique site identifier & \\
\hline & $\begin{array}{l}\text { Plot } \\
\text { identification }\end{array}$ & $\begin{array}{l}\text { Unique plot } \\
\text { identifier }\end{array}$ & \\
\hline & Coordinates & Latitude/longitude & $\begin{array}{l}\text { Specific to coordinate system, } \\
\text { deviation north }\end{array}$ \\
\hline & Plot shape & & \\
\hline & Contact & & \\
\hline \multirow{5}{*}{$\begin{array}{l}\text { Topographic } \\
\text { information }\end{array}$} & Aspect & & Degree, letters \\
\hline & Slope $\left(^{\circ}\right)$ & & Degree \\
\hline & $\begin{array}{l}\text { Position in the } \\
\text { slope }\end{array}$ & & Category \\
\hline & Slope length & & \\
\hline & $\begin{array}{l}\text { Elevation } \\
\text { (m a.s.1.) }\end{array}$ & & $\mathrm{m}$ a.s.l. \\
\hline \multirow[t]{3}{*}{$\begin{array}{l}\text { Information on soil } \\
\text { and climate }\end{array}$} & Parental material & $\begin{array}{l}\text { International } \\
\text { nomenclature }\end{array}$ & \\
\hline & Soil depth & & \\
\hline & Climate data & & \\
\hline \multirow[t]{2}{*}{ Other specifics } & Stand age & Estimates, inventory & Year \\
\hline & Further remarks & & \\
\hline
\end{tabular}

Table 5.3 Variables of single-tree measurements, exemplary for study 2

\begin{tabular}{l|l|l}
\hline Variable & Unit/number & Sample size (CLIMO study) \\
\hline Date of survey & $\begin{array}{l}1-899 \text { for inner plot } \\
\text { trees, } \geq 900 \text { for } \\
\text { border tree }\end{array}$ & Full inventory \\
\hline Tree coordinates & $\begin{array}{l}\text { Local } \mathrm{X}, \mathrm{Y} \\
\text { coordinates in } \mathrm{m}\end{array}$ & Full inventory \\
\hline $\begin{array}{l}\text { Diameter at breast } \\
\text { height }\end{array}$ & cm (one decimal) & Full inventory \\
\hline Tree height & $\mathrm{m}$ (one decimal) & Full inventory \\
\hline Height of crown base & $\mathrm{m}$ (one decimal) & Full inventory \\
\hline Crown radii & $\mathrm{m}$ (one decimal) & Full inventory, min. 4 cardinal directions \\
\hline $\begin{array}{l}\text { Specifics remarks } \\
\text { (e.g. damages, sample } \\
\text { tree for coring) }\end{array}$ & $\begin{array}{l}\text { Descriptive } \\
\text { (character type) }\end{array}$ & Specific trees \\
\hline Tree cores & $\begin{array}{l}2 \text { per tree, } \\
\text { perpendicular at } \\
1.3 \text { m }\end{array}$ & $\begin{array}{l}15 \text { dominant beeches, 15 trees covering the rest } \\
\text { of the diameter distribution, in case of mixed } \\
\text { stands } 15 \text { trees of mixed species covering the } \\
\text { diameter distribution }\end{array}$ \\
\hline
\end{tabular}


Table 5.4 Geographical information and site characteristics of the 159 observational plots

\begin{tabular}{|c|c|c|c|c|c|c|c|}
\hline \multirow[t]{2}{*}{ Country } & \multirow[t]{2}{*}{ Period } & \multirow[t]{2}{*}{ Composition } & \multicolumn{2}{|l|}{ Coordinates } & \multicolumn{3}{|c|}{$\begin{array}{l}\text { Site } \\
\text { characteristics }\end{array}$} \\
\hline & & & Latitude & Longitude & $\mathrm{E}$ & $\mathrm{T}$ & $\mathrm{P}$ \\
\hline $\begin{array}{l}\text { Bosnia and } \\
\text { Herzegovina }\end{array}$ & Long-term & Mixed & $43^{\circ} 47^{\prime} 54.6^{\prime \prime} \mathrm{N}$ & $18^{\circ} 16^{\prime} 49.6^{\prime \prime} \mathrm{E}$ & 1006 & 7.7 & 1179 \\
\hline $\begin{array}{l}\text { Bosnia and } \\
\text { Herzegovina }\end{array}$ & Long-term & Mixed & $43^{\circ} 45^{\prime} 18^{\prime \prime} \mathrm{N}$ & $18^{\circ} 18^{\prime} 11.2^{\prime \prime} \mathrm{E}$ & 1257 & 6.7 & 1333 \\
\hline $\begin{array}{l}\text { Bosnia and } \\
\text { Herzegovina }\end{array}$ & Long-term & Mixed & $43^{\circ} 44^{\prime} 55.8^{\prime \prime} \mathrm{N}$ & $18^{\circ} 15^{\prime} 03.2^{\prime \prime} \mathrm{E}$ & 1291 & 6.6 & 1354 \\
\hline $\begin{array}{l}\text { Bosnia and } \\
\text { Herzegovina }\end{array}$ & Long-term & Mixed & $43^{\circ} 44^{\prime} 49.1^{\prime \prime} \mathrm{N}$ & $18^{\circ} 15^{\prime} 54^{\prime \prime} \mathrm{E}$ & 1192 & 7.0 & 1293 \\
\hline $\begin{array}{l}\text { Bosnia and } \\
\text { Herzegovina }\end{array}$ & Long-term & Mixed & $43^{\circ} 46^{\prime} 27^{\prime \prime} \mathrm{N}$ & $18^{\circ} 17^{\prime} 54.4^{\prime \prime} \mathrm{E}$ & 1166 & 7.0 & 1277 \\
\hline Bulgaria & Long-term & Mixed & $41^{\circ} 55^{\prime} 06.8^{\prime \prime} \mathrm{N}$ & $23^{\circ} 50^{\prime} 29.7^{\prime \prime} \mathrm{E}$ & 1569 & 2.6 & 1066 \\
\hline Bulgaria & Long-term & Mixed & $41^{\circ} 57^{\prime} 55.6^{\prime \prime} \mathrm{N}$ & $24^{\circ} 31^{\prime} 14^{\prime \prime} \mathrm{E}$ & 1391 & 3.3 & 956 \\
\hline Germany & Long-term & Mixed & $47^{\circ} 43^{\prime} 47.6^{\prime \prime} \mathrm{N}$ & $10^{\circ} 32^{\prime} 24.9^{\prime \prime} \mathrm{E}$ & 826 & 7.2 & 1426 \\
\hline Germany & Long-term & Mixed & $47^{\circ} 37^{\prime} 19.6^{\prime \prime} \mathrm{N}$ & $11^{\circ} 53^{\prime} 59.8^{\prime \prime} \mathrm{E}$ & 1136 & 6.6 & 1615 \\
\hline Germany & Long-term & Mixed & $47^{\circ} 35^{\prime} 38.2^{\prime \prime} \mathrm{N}$ & $11^{\circ} 41^{\prime} 41.1^{\prime \prime} \mathrm{E}$ & 1271 & 4.7 & 2281 \\
\hline Germany & Long-term & Mixed & $47^{\circ} 45^{\prime} 51.3^{\prime \prime} \mathrm{N}$ & $12^{\circ} 29^{\prime} 44.8^{\prime \prime} \mathrm{E}$ & 939 & 5.8 & 1936 \\
\hline Germany & Long-term & Mixed & $47^{\circ} 42^{\prime} 14.4^{\prime \prime} \mathrm{N}$ & $12^{\circ} 26^{\prime} 47.9^{\prime \prime} \mathrm{E}$ & 927 & 5.1 & 2000 \\
\hline Germany & Long-term & Mixed & $47^{\circ} 42^{\prime} 12.9^{\prime \prime} \mathrm{N}$ & $12^{\circ} 28^{\prime} 26.3^{\prime \prime} \mathrm{E}$ & 860 & 6.8 & 1646 \\
\hline Germany & Long-term & Mixed & $47^{\circ} 26^{\prime} 15.7^{\prime \prime} \mathrm{N}$ & $11^{\circ} 09^{\prime} 57.3^{\prime \prime} \mathrm{E}$ & 1463 & 4.5 & 1745 \\
\hline Germany & Long-term & Mixed & $47^{\circ} 25^{\prime} 59.8^{\prime \prime} \mathrm{N}$ & $11^{\circ} 09^{\prime} 48.5^{\prime \prime} \mathrm{E}$ & 1768 & 2.0 & 2182 \\
\hline Germany & Long-term & Mixed & $47^{\circ} 44^{\prime} 10.6^{\prime \prime} \mathrm{N}$ & $12^{\circ} 21^{\prime} 51.4^{\prime \prime} \mathrm{E}$ & 902 & 5.1 & 2236 \\
\hline Germany & Long-term & Mixed & $47^{\circ} 43^{\prime} 02.2^{\prime \prime} \mathrm{N}$ & $12^{\circ} 42^{\prime} 15^{\prime \prime} \mathrm{E}$ & 934 & 6.1 & 1810 \\
\hline Germany & Long-term & Mixed & $47^{\circ} 43^{\prime} 02.2^{\prime \prime} \mathrm{N}$ & $12^{\circ} 42^{\prime} 15^{\prime \prime} \mathrm{E}$ & 934 & 6.1 & 1810 \\
\hline Germany & Long-term & Mixed & $47^{\circ} 42^{\prime} 50.1^{\prime \prime} \mathrm{N}$ & $12^{\circ} 42^{\prime} 27.3^{\prime \prime} \mathrm{E}$ & 973 & 6.1 & 1810 \\
\hline Germany & Long-term & Mixed & $47^{\circ} 42^{\prime} 50.1^{\prime \prime} \mathrm{N}$ & $12^{\circ} 42^{\prime} 27.3^{\prime \prime} \mathrm{E}$ & 973 & 6.1 & 1810 \\
\hline Germany & Long-term & Mixed & $47^{\circ} 26^{\prime} 52.2^{\prime \prime} \mathrm{N}$ & $11^{\circ} 07^{\prime} 24.6^{\prime \prime} \mathrm{E}$ & 1235 & 4.8 & 1470 \\
\hline Germany & Long-term & Mixed & $47^{\circ} 26^{\prime} 52.2^{\prime \prime} \mathrm{N}$ & $11^{\circ} 07^{\prime} 24.6^{\prime \prime} \mathrm{E}$ & 1235 & 4.8 & 1470 \\
\hline Germany & Long-term & Mixed & $47^{\circ} 26^{\prime} 52.2^{\prime \prime} \mathrm{N}$ & $11^{\circ} 07^{\prime} 24.6^{\prime \prime} \mathrm{E}$ & 1235 & 4.8 & 1470 \\
\hline Germany & Long-term & Mixed & $47^{\circ} 26^{\prime} 52.2^{\prime \prime} \mathrm{N}$ & $11^{\circ} 07^{\prime} 24.6^{\prime \prime} \mathrm{E}$ & 1235 & 4.8 & 1470 \\
\hline Germany & Long-term & Mixed & $47^{\circ} 42^{\prime} 56.6^{\prime \prime} \mathrm{N}$ & $12^{\circ} 40^{\prime} 09.7^{\prime \prime} \mathrm{E}$ & 884 & 6.8 & 1707 \\
\hline Germany & Long-term & Mixed & $47^{\circ} 36^{\prime} 04^{\prime \prime} \mathrm{N}$ & $11^{\circ} 39^{\prime} 43.9^{\prime \prime} \mathrm{E}$ & 1091 & 6.1 & 1998 \\
\hline Germany & Long-term & Mixed & $47^{\circ} 36^{\prime} 04^{\prime \prime} \mathrm{N}$ & $11^{\circ} 39^{\prime} 43.9^{\prime \prime} \mathrm{E}$ & 1091 & 6.1 & 1998 \\
\hline Germany & Long-term & Mixed & $47^{\circ} 36^{\prime} 04^{\prime \prime} \mathrm{N}$ & $11^{\circ} 39^{\prime} 43.9^{\prime \prime} \mathrm{E}$ & 1091 & 6.1 & 1998 \\
\hline Germany & Long-term & Mixed & $47^{\circ} 36^{\prime} 04^{\prime \prime} \mathrm{N}$ & $11^{\circ} 39^{\prime} 43.9^{\prime \prime} \mathrm{E}$ & 1091 & 6.1 & 1998 \\
\hline Germany & Long-term & Mixed & $47^{\circ} 39^{\prime} 18.1^{\prime \prime} \mathrm{N}$ & $11^{\circ} 43^{\prime} 13.3^{\prime \prime} \mathrm{E}$ & 1281 & 6.1 & 2059 \\
\hline Germany & Long-term & Mixed & $47^{\circ} 39^{\prime} 18.1^{\prime \prime} \mathrm{N}$ & $11^{\circ} 43^{\prime} 13.3^{\prime \prime} \mathrm{E}$ & 1281 & 6.1 & 2059 \\
\hline Germany & Long-term & Mixed & $47^{\circ} 39^{\prime} 18.1^{\prime \prime} \mathrm{N}$ & $11^{\circ} 43^{\prime} 13.3^{\prime \prime} \mathrm{E}$ & 1281 & 6.1 & 2059 \\
\hline Germany & Long-term & Mixed & $47^{\circ} 39^{\prime} 18.1^{\prime \prime} \mathrm{N}$ & $11^{\circ} 43^{\prime} 13.3^{\prime \prime} \mathrm{E}$ & 1281 & 6.1 & 2059 \\
\hline Germany & Long-term & Mixed & $48^{\circ} 51^{\prime} 19.2^{\prime \prime} \mathrm{N}$ & $13^{\circ} 35^{\prime} 18.4^{\prime \prime} \mathrm{E}$ & 743 & 6.8 & 1072 \\
\hline
\end{tabular}


Table 5.4 (continued)

\begin{tabular}{|c|c|c|c|c|c|c|c|}
\hline \multirow[t]{2}{*}{ Country } & \multirow[t]{2}{*}{ Period } & \multirow[t]{2}{*}{ Composition } & \multicolumn{2}{|l|}{ Coordinates } & \multicolumn{3}{|c|}{$\begin{array}{l}\text { Site } \\
\text { characteristics }\end{array}$} \\
\hline & & & Latitude & Longitude & $\mathrm{E}$ & $\mathrm{T}$ & $\mathrm{P}$ \\
\hline Germany & Long-term & Mixed & $48^{\circ} 51^{\prime} 19.2^{\prime \prime} \mathrm{N}$ & $13^{\circ} 35^{\prime} 18.4^{\prime \prime} \mathrm{E}$ & 743 & 6.8 & 1072 \\
\hline Germany & Long-term & Mixed & $48^{\circ} 51^{\prime} 19.2^{\prime \prime} \mathrm{N}$ & $13^{\circ} 35^{\prime} 18.4^{\prime \prime} \mathrm{E}$ & 743 & 6.8 & 1072 \\
\hline Germany & Long-term & Mixed & $48^{\circ} 51^{\prime} 19.2^{\prime \prime} \mathrm{N}$ & $13^{\circ} 35^{\prime} 18.4^{\prime \prime} \mathrm{E}$ & 743 & 6.8 & 1072 \\
\hline Germany & Long-term & Mixed & $48^{\circ} 51^{\prime} 19.2^{\prime \prime} \mathrm{N}$ & $13^{\circ} 35^{\prime} 18.4^{\prime \prime} \mathrm{E}$ & 743 & 6.8 & 1072 \\
\hline Germany & Long-term & Mixed & $48^{\circ} 51^{\prime} 19.2^{\prime \prime} \mathrm{N}$ & $13^{\circ} 35^{\prime} 18.4^{\prime \prime} \mathrm{E}$ & 743 & 6.8 & 1072 \\
\hline Germany & Long-term & Mixed & $49^{\circ} 05^{\prime} 55.1^{\prime \prime} \mathrm{N}$ & $13^{\circ} 05^{\prime} 30.1^{\prime \prime} \mathrm{E}$ & 951 & 5.2 & 1339 \\
\hline Germany & Long-term & Mixed & $49^{\circ} 05^{\prime} 55.1^{\prime \prime} \mathrm{N}$ & $13^{\circ} 05^{\prime} 30.1^{\prime \prime} \mathrm{E}$ & 951 & 5.2 & 1339 \\
\hline Germany & Long-term & Mixed & $49^{\circ} 05^{\prime} 55.1^{\prime \prime} \mathrm{N}$ & $13^{\circ} 05^{\prime} 30.1^{\prime \prime} \mathrm{E}$ & 951 & 5.2 & 1339 \\
\hline Germany & Long-term & Mixed & $49^{\circ} 05^{\prime} 55.1^{\prime \prime} \mathrm{N}$ & $13^{\circ} 05^{\prime} 30.1^{\prime \prime} \mathrm{E}$ & 951 & 5.2 & 1339 \\
\hline Germany & Long-term & Mixed & $49^{\circ} 05^{\prime} 18.9^{\prime \prime} \mathrm{N}$ & $13^{\circ} 17^{\prime} 41.7^{\prime \prime} \mathrm{E}$ & 1037 & 4.3 & 1402 \\
\hline Germany & Long-term & Mixed & $49^{\circ} 05^{\prime} 18.9^{\prime \prime} \mathrm{N}$ & $13^{\circ} 17^{\prime} 41.7^{\prime \prime} \mathrm{E}$ & 1037 & 4.3 & 1402 \\
\hline Germany & Long-term & Mixed & $49^{\circ} 05^{\prime} 53.7^{\prime \prime} \mathrm{N}$ & $13^{\circ} 15^{\prime} 07.1^{\prime \prime} \mathrm{E}$ & 787 & 5.8 & 1344 \\
\hline Germany & Long-term & Mixed & $49^{\circ} 05^{\prime} 59.4^{\prime \prime} \mathrm{N}$ & $13^{\circ} 14^{\prime} 59^{\prime \prime} \mathrm{E}$ & 779 & 6.5 & 1294 \\
\hline Germany & Long-term & Mixed & $47^{\circ} 37^{\prime} 57.5^{\prime \prime} \mathrm{N}$ & $11^{\circ} 41^{\prime} 23.2^{\prime \prime} \mathrm{E}$ & 1294 & 5.4 & 2163 \\
\hline Poland & Long-term & Mixed & $50^{\circ} 53^{\prime} 39.5^{\prime \prime} \mathrm{N}$ & $20^{\circ} 54^{\prime} 09.5^{\prime \prime} \mathrm{E}$ & 501 & 6.5 & 731 \\
\hline Poland & Long-term & Mixed & $50^{\circ} 53^{\prime} 25.6^{\prime \prime} \mathrm{N}$ & $20^{\circ} 53^{\prime} 56^{\prime \prime} \mathrm{E}$ & 600 & 6.1 & 791 \\
\hline Poland & Long-term & Mixed & $50^{\circ} 53^{\prime} 52.1^{\prime \prime} \mathrm{N}$ & $20^{\circ} 54^{\prime} 22.3^{\prime \prime} \mathrm{E}$ & 425 & 6.8 & 684 \\
\hline Poland & Long-term & Mixed & $49^{\circ} 35^{\prime} 38.9^{\prime \prime} \mathrm{N}$ & $19^{\circ} 28^{\prime} 42.6^{\prime \prime} \mathrm{E}$ & 1015 & 5.0 & 1403 \\
\hline Poland & Long-term & Mixed & $49^{\circ} 35^{\prime} 36.7^{\prime \prime} \mathrm{N}$ & $19^{\circ} 33^{\prime} 24.2^{\prime \prime} \mathrm{E}$ & 972 & 5.2 & 1377 \\
\hline Poland & Long-term & Mixed & $49^{\circ} 35^{\prime} 36^{\prime \prime} \mathrm{N}$ & $19^{\circ} 33^{\prime} 12.1^{\prime \prime} \mathrm{E}$ & 966 & 5.2 & 1373 \\
\hline Poland & Long-term & Mixed & $49^{\circ} 35^{\prime} 50.7^{\prime \prime} \mathrm{N}$ & $19^{\circ} 28^{\prime} 36.6^{\prime \prime} \mathrm{E}$ & 902 & 5.5 & 1334 \\
\hline Poland & Long-term & Mixed & $49^{\circ} 35^{\prime} 35.5^{\prime \prime} \mathrm{N}$ & $19^{\circ} 33^{\prime} 39.2^{\prime \prime} \mathrm{E}$ & 982 & 5.2 & 1383 \\
\hline Poland & Long-term & Mixed & $49^{\circ} 35^{\prime} 37.6^{\prime \prime} \mathrm{N}$ & $19^{\circ} 33^{\prime} 42.7^{\prime \prime} \mathrm{E}$ & 958 & 5.3 & 1368 \\
\hline Poland & Long-term & Mixed & $49^{\circ} 35^{\prime} 24.6^{\prime \prime} \mathrm{N}$ & $19^{\circ} 34^{\prime} 07.2^{\prime \prime} \mathrm{E}$ & 1087 & 4.8 & 1447 \\
\hline Slovakia & Long-term & Mixed & $48^{\circ} 38^{\prime} 34.1^{\prime \prime} \mathrm{N}$ & $19^{\circ} 32^{\prime} 21.8^{\prime \prime} \mathrm{E}$ & 803 & 5.5 & 780 \\
\hline Slovakia & Long-term & Mixed & $48^{\circ} 46^{\prime} 22.1^{\prime \prime} \mathrm{N}$ & $20^{\circ} 44^{\prime} 36.3^{\prime \prime} \mathrm{E}$ & 773 & 5.6 & 862 \\
\hline Slovakia & Long-term & Mixed & $48^{\circ} 46^{\prime} 18.8^{\prime \prime} \mathrm{N}$ & $20^{\circ} 43^{\prime} 32.3^{\prime \prime} \mathrm{E}$ & 738 & 5.7 & 840 \\
\hline Slovakia & Long-term & Mixed & $48^{\circ} 47^{\prime} 23.8^{\prime \prime} \mathrm{N}$ & $20^{\circ} 40^{\prime} 07.3^{\prime \prime} \mathrm{E}$ & 621 & 6.2 & 769 \\
\hline Slovakia & Long-term & Mixed & $48^{\circ} 45^{\prime} 35.1^{\prime \prime} \mathrm{N}$ & $20^{\circ} 42^{\prime} 56.9^{\prime \prime} \mathrm{E}$ & 845 & 5.3 & 906 \\
\hline Slovakia & Long-term & Mixed & $48^{\circ} 36^{\prime} 57^{\prime \prime} \mathrm{N}$ & $19^{\circ} 33^{\prime} 57.6^{\prime \prime} \mathrm{E}$ & 693 & 6.6 & 796 \\
\hline Slovakia & Long-term & Mixed & $48^{\circ} 37^{\prime} 26.1^{\prime \prime} \mathrm{N}$ & $19^{\circ} 35^{\prime} 59.9^{\prime \prime} \mathrm{E}$ & 786 & 6.2 & 854 \\
\hline Slovakia & Long-term & Mixed & $48^{\circ} 37^{\prime} 55.6^{\prime \prime} \mathrm{N}$ & $19^{\circ} 34^{\prime} 17.4^{\prime \prime} \mathrm{E}$ & 733 & 6.4 & 821 \\
\hline Slovenia & Long-term & Mixed & $45^{\circ} 45^{\prime} 13.7^{\prime \prime} \mathrm{N}$ & $14^{\circ} 59^{\prime} 42.2^{\prime \prime} \mathrm{E}$ & 909 & 6.9 & 1751 \\
\hline Slovenia & Long-term & Mixed & $45^{\circ} 45^{\prime} 13.7^{\prime \prime} \mathrm{N}$ & $14^{\circ} 59^{\prime} 42.2^{\prime \prime} \mathrm{E}$ & 909 & 6.9 & 1751 \\
\hline Slovenia & Long-term & Mixed & $45^{\circ} 45^{\prime} 13.7^{\prime \prime} \mathrm{N}$ & $14^{\circ} 59^{\prime} 42.2^{\prime \prime} \mathrm{E}$ & 909 & 6.9 & 1751 \\
\hline Slovenia & Long-term & Mixed & $45^{\circ} 39^{\prime} 51.8^{\prime \prime} \mathrm{N}$ & $15^{\circ} 00^{\prime} 25.3^{\prime \prime} \mathrm{E}$ & 910 & 6.9 & 1756 \\
\hline Slovenia & Long-term & Mixed & $45^{\circ} 39^{\prime} 51.8^{\prime \prime} \mathrm{N}$ & $15^{\circ} 00^{\prime} 25.3^{\prime \prime} \mathrm{E}$ & 910 & 6.9 & 1756 \\
\hline
\end{tabular}


Table 5.4 (continued)

\begin{tabular}{|c|c|c|c|c|c|c|c|}
\hline \multirow[t]{2}{*}{ Country } & \multirow[t]{2}{*}{ Period } & \multirow[t]{2}{*}{ Composition } & \multicolumn{2}{|l|}{ Coordinates } & \multicolumn{3}{|c|}{$\begin{array}{l}\text { Site } \\
\text { characteristics }\end{array}$} \\
\hline & & & Latitude & Longitude & $\mathrm{E}$ & $\mathrm{T}$ & $\mathrm{P}$ \\
\hline Slovenia & Long-term & Mixed & $45^{\circ} 39^{\prime} 51.8^{\prime \prime} \mathrm{N}$ & $15^{\circ} 00^{\prime} 25.3^{\prime \prime} \mathrm{E}$ & 910 & 6.9 & 1756 \\
\hline Slovenia & Long-term & Mixed & $45^{\circ} 37^{\prime} 21.4^{\prime \prime} \mathrm{N}$ & $14^{\circ} 48^{\prime} 52.9^{\prime \prime} \mathrm{E}$ & 917 & 6.9 & 1757 \\
\hline Slovenia & Long-term & Mixed & $46^{\circ} 29^{\prime} 14.3^{\prime \prime} \mathrm{N}$ & $15^{\circ} 27^{\prime} 18.5^{\prime \prime} \mathrm{E}$ & 970 & 6.0 & 1464 \\
\hline Slovenia & Long-term & Mixed & $46^{\circ} 14^{\prime} 49.6^{\prime \prime} \mathrm{N}$ & $14^{\circ} 03^{\prime} 40.3^{\prime \prime} \mathrm{E}$ & 1426 & 4.7 & 2770 \\
\hline Slovenia & Long-term & Mixed & $46^{\circ} 14^{\prime} 56^{\prime \prime} \mathrm{N}$ & $14^{\circ} 03^{\prime} 40.2^{\prime \prime} \mathrm{E}$ & 1375 & 4.9 & 2738 \\
\hline Slovenia & Long-term & Mixed & $46^{\circ} 14^{\prime} 55.6^{\prime \prime} \mathrm{N}$ & $14^{\circ} 02^{\prime} 44.1^{\prime \prime} \mathrm{E}$ & 1443 & 4.7 & 2780 \\
\hline Slovenia & Long-term & Mixed & $46^{\circ} 15^{\prime} 02.5^{\prime \prime} \mathrm{N}$ & $14^{\circ} 02^{\prime} 43.9^{\prime \prime} \mathrm{E}$ & 1421 & 4.7 & 2767 \\
\hline Slovenia & Long-term & Mixed & $46^{\circ} 15^{\prime} 08.5^{\prime \prime} \mathrm{N}$ & $14^{\circ} 02^{\prime} 34.8^{\prime \prime} \mathrm{E}$ & 1375 & 4.9 & 2738 \\
\hline Switzerland & Long-term & Mixed & $46^{\circ} 52^{\prime} 33.5^{\prime \prime} \mathrm{N}$ & $7^{\circ} 41^{\prime} 14.9^{\prime \prime} \mathrm{E}$ & 899 & 7.2 & 1390 \\
\hline Switzerland & Long-term & Mixed & $46^{\circ} 52^{\prime} 33.5^{\prime \prime} \mathrm{N}$ & $7^{\circ} 41^{\prime} 14.9^{\prime \prime} \mathrm{E}$ & 899 & 7.2 & 1390 \\
\hline Switzerland & Long-term & Mixed & $46^{\circ} 52^{\prime} 33.5^{\prime \prime} \mathrm{N}$ & $7^{\circ} 41^{\prime} 14.9^{\prime \prime} \mathrm{E}$ & 899 & 7.2 & 1390 \\
\hline Switzerland & Long-term & Mixed & $46^{\circ} 57^{\prime} 34.5^{\prime \prime} \mathrm{N}$ & $7^{\circ} 46^{\prime} 25.2^{\prime \prime} \mathrm{E}$ & 890 & 7.1 & 1448 \\
\hline Switzerland & Long-term & Mixed & $46^{\circ} 57^{\prime} 34.5^{\prime \prime} \mathrm{N}$ & $7^{\circ} 46^{\prime} 25.2^{\prime \prime} \mathrm{E}$ & 890 & 7.1 & 1448 \\
\hline Switzerland & Long-term & Mixed & $47^{\circ} 20^{\prime} 05.4^{\prime \prime} \mathrm{N}$ & $7^{\circ} 09^{\prime} 53.1^{\prime \prime} \mathrm{E}$ & 790 & 7.4 & 1302 \\
\hline Switzerland & Long-term & Mixed & $47^{\circ} 20^{\prime} 15^{\prime \prime} \mathrm{N}$ & $7^{\circ} 09^{\prime} 05.4^{\prime \prime} \mathrm{E}$ & 558 & 8.8 & 1140 \\
\hline Switzerland & Long-term & Mixed & $47^{\circ} 20^{\prime} 15^{\prime \prime} \mathrm{N}$ & $7^{\circ} 09^{\prime} 05.4^{\prime \prime} \mathrm{E}$ & 558 & 8.8 & 1140 \\
\hline Switzerland & Long-term & Mixed & $46^{\circ} 56^{\prime} 45.6^{\prime \prime} \mathrm{N}$ & $7^{\circ} 39^{\prime} 42.5^{\prime \prime} \mathrm{E}$ & 981 & 6.7 & 1477 \\
\hline Switzerland & Long-term & Mixed & $46^{\circ} 33^{\prime} 31.8^{\prime \prime} \mathrm{N}$ & $6^{\circ} 13^{\prime} 18.8^{\prime \prime} \mathrm{E}$ & 1364 & 4.8 & 1796 \\
\hline $\begin{array}{l}\text { Bosnia and } \\
\text { Herzegovina }\end{array}$ & Temporary & Mixed & $44^{\circ} 38^{\prime} 30^{\prime \prime} \mathrm{N}$ & $16^{\circ} 39^{\prime} 36.1^{\prime \prime} \mathrm{E}$ & 725 & 11.6 & 937 \\
\hline $\begin{array}{l}\text { Bosnia and } \\
\text { Herzegovina }\end{array}$ & Temporary & Mixed & $43^{\circ} 43^{\prime} 28^{\prime \prime} \mathrm{N}$ & $18^{\circ} 17^{\prime} 09^{\prime \prime} \mathrm{E}$ & 1300 & 8.3 & 992 \\
\hline $\begin{array}{l}\text { Bosnia and } \\
\text { Herzegovina }\end{array}$ & Temporary & Mixed & $44^{\circ} 41^{\prime} 09.1^{\prime \prime} \mathrm{N}$ & $16^{\circ} 29^{\prime} 40.5^{\prime \prime} \mathrm{E}$ & 663 & 11.4 & 1028 \\
\hline Germany & Temporary & Mixed & $49^{\circ} 05^{\prime} 08.4^{\prime \prime} \mathrm{N}$ & $13^{\circ} 18^{\prime} 23.5^{\prime \prime} \mathrm{E}$ & 1120 & 6.5 & 1078 \\
\hline Italy & Temporary & Mixed & $41^{\circ} 52^{\prime} 14.4^{\prime \prime} \mathrm{N}$ & $14^{\circ} 16^{\prime} 51.3^{\prime \prime} \mathrm{E}$ & 1332 & 11.3 & 692 \\
\hline Italy & Temporary & Mixed & $39^{\circ} 09^{\prime} 13.5^{\prime \prime} \mathrm{N}$ & $16^{\circ} 39^{\prime} 53^{\prime \prime} \mathrm{E}$ & 1182 & 11.3 & 969 \\
\hline Italy & Temporary & Mixed & $46^{\circ} 12^{\prime} 06.4^{\prime \prime} \mathrm{N}$ & $11^{\circ} 12^{\prime} 36.9^{\prime \prime} \mathrm{E}$ & 1271 & 9.1 & 932 \\
\hline Italy & Temporary & Mixed & $46^{\circ} 05^{\prime} 56^{\prime \prime} \mathrm{N}$ & $12^{\circ} 25^{\prime} 49^{\prime \prime} \mathrm{E}$ & 1100 & 8.0 & 1057 \\
\hline Italy & Temporary & Mixed & $41^{\circ} 52^{\prime} 11^{\prime \prime} \mathrm{N}$ & $14^{\circ} 17^{\prime} 26^{\prime \prime} \mathrm{E}$ & 1289 & 11.0 & 692 \\
\hline Poland & Temporary & Mixed & $49^{\circ} 37^{\prime} 10.3^{\prime \prime} \mathrm{N}$ & $18^{\circ} 55^{\prime} 07.5^{\prime \prime} \mathrm{E}$ & 528 & 9.0 & 1128 \\
\hline Poland & Temporary & Mixed & $49^{\circ} 37^{\prime} 33.2^{\prime \prime} \mathrm{N}$ & $18^{\circ} 55^{\prime} 11.8^{\prime \prime} \mathrm{E}$ & 665 & 8.2 & 1128 \\
\hline Romania & Temporary & Mixed & $45^{\circ} 32^{\prime} 15.3^{\prime \prime} \mathrm{N}$ & $25^{\circ} 52^{\prime} 51.2^{\prime \prime} \mathrm{E}$ & 1251 & 6.4 & 624 \\
\hline Serbia & Temporary & Mixed & $43^{\circ} 19^{\prime} 06.1^{\prime \prime} \mathrm{N}$ & $19^{\circ} 51^{\prime} 50.6^{\prime \prime} \mathrm{E}$ & 1227 & 8.1 & 823 \\
\hline Serbia & Temporary & Mixed & $43^{\circ} 24^{\prime} 22.3^{\prime \prime} \mathrm{N}$ & $21^{\circ} 22^{\prime} 41.1^{\prime \prime} \mathrm{E}$ & 691 & 8.7 & 668 \\
\hline Serbia & Temporary & Mixed & $43^{\circ} 29^{\prime} 12^{\prime \prime} \mathrm{N}$ & $19^{\circ} 51^{\prime} 38^{\prime \prime} \mathrm{E}$ & 1221 & 8.2 & 839 \\
\hline Serbia & Temporary & Mixed & $43^{\circ} 21^{\prime} 01.8^{\prime \prime} \mathrm{N}$ & $20^{\circ} 15^{\prime} 17.6^{\prime \prime} \mathrm{E}$ & 1470 & 6.7 & 821 \\
\hline Slovakia & Temporary & Mixed & $48^{\circ} 40^{\prime} 40.7^{\prime \prime} \mathrm{N}$ & $19^{\circ} 28^{\prime} 12.6^{\prime \prime} \mathrm{E}$ & 1180 & 6.1 & 889 \\
\hline
\end{tabular}


Table 5.4 (continued)

\begin{tabular}{|c|c|c|c|c|c|c|c|}
\hline \multirow[t]{2}{*}{ Country } & \multirow[t]{2}{*}{ Period } & \multirow[t]{2}{*}{ Composition } & \multicolumn{2}{|l|}{ Coordinates } & \multicolumn{3}{|c|}{$\begin{array}{l}\text { Site } \\
\text { characteristics }\end{array}$} \\
\hline & & & Latitude & Longitude & $\mathrm{E}$ & $\mathrm{T}$ & $\mathrm{P}$ \\
\hline Slovenia & Temporary & Mixed & $46^{\circ} 05^{\prime} 33.7^{\prime \prime} \mathrm{N}$ & $15^{\circ} 03^{\prime} 44.3^{\prime \prime} \mathrm{E}$ & 1030 & 8.4 & 1223 \\
\hline Spain & Temporary & Mixed & $42^{\circ} 16^{\prime} 11.4^{\prime \prime} \mathrm{N}$ & $3^{\circ} 16^{\prime} 05.4^{\prime \prime} \mathrm{W}$ & 1525 & 9.5 & 631 \\
\hline Spain & Temporary & Mixed & $42^{\circ} 12^{\prime} 03^{\prime \prime} \mathrm{N}$ & $2^{\circ} 43^{\prime} 07^{\prime \prime} \mathrm{W}$ & 1390 & 10.7 & 575 \\
\hline Ukraine & Temporary & Mixed & $49^{\circ} 01^{\prime} 09^{\prime \prime} \mathrm{N}$ & $23^{\circ} 28^{\prime} 10^{\prime \prime} \mathrm{E}$ & 763 & 7.9 & 965 \\
\hline Ukraine & Temporary & Mixed & $48^{\circ} 51^{\prime} 12^{\prime \prime} \mathrm{N}$ & $22^{\circ} 58^{\prime} 60^{\prime \prime} \mathrm{E}$ & 1084 & 6.4 & 1063 \\
\hline $\begin{array}{l}\text { Bosnia and } \\
\text { Herzegovina }\end{array}$ & Temporary & Monospecific & $44^{\circ} 38^{\prime} 38.7^{\prime \prime} \mathrm{N}$ & $16^{\circ} 40^{\prime} 06.4^{\prime \prime} \mathrm{E}$ & 524 & 12.7 & 937 \\
\hline $\begin{array}{l}\text { Bosnia and } \\
\text { Herzegovina }\end{array}$ & Temporary & Monospecific & $44^{\circ} 41^{\prime} 07^{\prime \prime} \mathrm{N}$ & $16^{\circ} 29^{\prime} 43.2^{\prime \prime} \mathrm{E}$ & 669 & 11.4 & 1028 \\
\hline $\begin{array}{l}\text { Bosnia and } \\
\text { Herzegovina }\end{array}$ & Temporary & Monospecific & $43^{\circ} 42^{\prime} 25^{\prime \prime} \mathrm{N}$ & $18^{\circ} 15^{\prime} 44^{\prime \prime} \mathrm{E}$ & 1292 & 8.4 & 992 \\
\hline $\begin{array}{l}\text { Bosnia and } \\
\text { Herzegovina }\end{array}$ & Temporary & Monospecific & $43^{\circ} 44^{\prime} 41^{\prime \prime} \mathrm{N}$ & $18^{\circ} 13^{\prime} 21^{\prime \prime} \mathrm{E}$ & 1680 & 6.1 & 1022 \\
\hline Bulgaria & Temporary & Monospecific & $42^{\circ} 40^{\prime} 21^{\prime \prime} \mathrm{N}$ & $23^{\circ} 51^{\prime} 03^{\prime \prime} \mathrm{E}$ & 1050 & 9.8 & 521 \\
\hline Bulgaria & Temporary & Monospecific & $42^{\circ} 46^{\prime} 45^{\prime \prime} \mathrm{N}$ & $23^{\circ} 52^{\prime} 52^{\prime \prime} \mathrm{E}$ & 1350 & 8.1 & 539 \\
\hline Bulgaria & Temporary & Monospecific & $42^{\circ} 40^{\prime} 23^{\prime \prime} \mathrm{N}$ & $23^{\circ} 51^{\prime} 07^{\prime \prime} \mathrm{E}$ & 1000 & 10.1 & 521 \\
\hline $\begin{array}{l}\text { Czech } \\
\text { Republic }\end{array}$ & Temporary & Monospecific & $49^{\circ} 17^{\prime} 06.6^{\prime \prime} \mathrm{N}$ & $16^{\circ} 44^{\prime} 21.4^{\prime \prime} \mathrm{E}$ & 490 & 9.6 & 517 \\
\hline $\begin{array}{l}\text { Czech } \\
\text { Republic }\end{array}$ & Temporary & Monospecific & $49^{\circ} 17^{\prime} 05.1^{\prime \prime} \mathrm{N}$ & $16^{\circ} 44^{\prime} 24.3^{\prime \prime} \mathrm{E}$ & 485 & 9.6 & 517 \\
\hline $\begin{array}{l}\text { Czech } \\
\text { Republic }\end{array}$ & Temporary & Monospecific & $49^{\circ} 10^{\prime} 17.3^{\prime \prime} \mathrm{N}$ & $19^{\circ} 04^{\prime} 54.5^{\prime \prime} \mathrm{E}$ & 767 & 7.7 & 1037 \\
\hline $\begin{array}{l}\text { Czech } \\
\text { Republic }\end{array}$ & Temporary & Monospecific & $49^{\circ} 10^{\prime} 53.1^{\prime \prime} \mathrm{N}$ & $19^{\circ} 05^{\prime} 40^{\prime \prime} \mathrm{E}$ & 1131 & 5.7 & 1037 \\
\hline $\begin{array}{l}\text { Czech } \\
\text { Republic }\end{array}$ & Temporary & Monospecific & $49^{\circ} 10^{\prime} 39^{\prime \prime} \mathrm{N}$ & $19^{\circ} 05^{\prime} 33.7^{\prime \prime} \mathrm{E}$ & 1146 & 5.8 & 1037 \\
\hline $\begin{array}{l}\text { Czech } \\
\text { Republic }\end{array}$ & Temporary & Monospecific & $49^{\circ} 02^{\prime} 08.3^{\prime \prime} \mathrm{N}$ & $18^{\circ} 01^{\prime} 07.5^{\prime \prime} \mathrm{E}$ & 415 & 10.0 & 753 \\
\hline $\begin{array}{l}\text { Czech } \\
\text { Republic }\end{array}$ & Temporary & Monospecific & $49^{\circ} 01^{\prime} 24.4^{\prime \prime} \mathrm{N}$ & $18^{\circ} 01^{\prime} 30.7^{\prime \prime} \mathrm{E}$ & 620 & 8.9 & 753 \\
\hline Germany & Temporary & Monospecific & $49^{\circ} 03^{\prime} 45.9^{\prime \prime} \mathrm{N}$ & $13^{\circ} 16^{\prime} 17.2^{\prime \prime} \mathrm{E}$ & 720 & 8.6 & 1078 \\
\hline Germany & Temporary & Monospecific & $49^{\circ} 03^{\prime} 49.3^{\prime \prime} \mathrm{N}$ & $13^{\circ} 16^{\prime} 06.1^{\prime \prime} \mathrm{E}$ & 695 & 8.7 & 1078 \\
\hline Hungary & Temporary & Monospecific & $47^{\circ} 21^{\prime} 46.3^{\prime \prime} \mathrm{N}$ & $16^{\circ} 29^{\prime} 12.2^{\prime \prime} \mathrm{E}$ & 640 & 10.0 & 602 \\
\hline Hungary & Temporary & Monospecific & $47^{\circ} 21^{\prime} 10.4^{\prime \prime} \mathrm{N}$ & $16^{\circ} 26^{\prime} 16.6^{\prime \prime} \mathrm{E}$ & 840 & 9.0 & 638 \\
\hline Italy & Temporary & Monospecific & $39^{\circ} 09^{\prime} 08^{\prime \prime} \mathrm{N}$ & $16^{\circ} 40^{\prime} 12.3^{\prime \prime} \mathrm{E}$ & 1182 & 11.1 & 969 \\
\hline Italy & Temporary & Monospecific & $45^{\circ} 57^{\prime} 43.7^{\prime \prime} \mathrm{N}$ & $11^{\circ} 16^{\prime} 26.6^{\prime \prime} \mathrm{E}$ & 1274 & 8.8 & 1064 \\
\hline Italy & Temporary & Monospecific & $46^{\circ} 07^{\prime} 08^{\prime \prime} \mathrm{N}$ & $12^{\circ} 25^{\prime} 47^{\prime \prime} \mathrm{E}$ & 1090 & 8.0 & 1057 \\
\hline Poland & Temporary & Monospecific & $49^{\circ} 37^{\prime} 20.8^{\prime \prime} \mathrm{N}$ & $18^{\circ} 54^{\prime} 52.6^{\prime \prime} \mathrm{E}$ & 520 & 9.0 & 1128 \\
\hline Poland & Temporary & Monospecific & $49^{\circ} 37^{\prime} 25.18^{\prime \prime} \mathrm{N}$ & $18^{\circ} 55^{\prime} 28.65^{\prime \prime} \mathrm{E}$ & 691 & 8.2 & 1128 \\
\hline Poland & Temporary & Monospecific & $49^{\circ} 25^{\prime} 58.7^{\prime \prime} \mathrm{N}$ & $20^{\circ} 54^{\prime} 11.2^{\prime \prime} \mathrm{E}$ & 830 & 7.7 & 814 \\
\hline
\end{tabular}


Table 5.4 (continued)

\begin{tabular}{|c|c|c|c|c|c|c|c|}
\hline \multirow[t]{2}{*}{ Country } & \multirow[t]{2}{*}{ Period } & \multirow[t]{2}{*}{ Composition } & \multicolumn{2}{|l|}{ Coordinates } & \multicolumn{3}{|c|}{$\begin{array}{l}\text { Site } \\
\text { characteristics }\end{array}$} \\
\hline & & & Latitude & Longitude & E & $\mathrm{T}$ & $\mathrm{P}$ \\
\hline Poland & Temporary & Monospecific & $49^{\circ} 25^{\prime} 35.5^{\prime \prime} \mathrm{N}$ & $20^{\circ} 53^{\prime} 56.8^{\prime \prime} \mathrm{E}$ & 860 & 7.6 & 814 \\
\hline Poland & Temporary & Monospecific & $49^{\circ} 26^{\prime} 01.8^{\prime \prime} \mathrm{N}$ & $20^{\circ} 52^{\prime} 46.4^{\prime \prime} \mathrm{E}$ & 1020 & 6.8 & 814 \\
\hline Poland & Temporary & Monospecific & $49^{\circ} 25^{\prime} 54.7^{\prime \prime} \mathrm{N}$ & $20^{\circ} 52^{\prime} 40.8^{\prime \prime} \mathrm{E}$ & 1032 & 6.7 & 814 \\
\hline Romania & Temporary & Monospecific & $45^{\circ} 19^{\prime} 15.3^{\prime \prime} \mathrm{N}$ & $25^{\circ} 48^{\prime} 19.3^{\prime \prime} \mathrm{E}$ & 970 & 8.1 & 588 \\
\hline Romania & Temporary & Monospecific & $45^{\circ} 32^{\prime} 14^{\prime \prime} \mathrm{N}$ & $25^{\circ} 53^{\prime} 01.7^{\prime \prime} \mathrm{E}$ & 1277 & 6.3 & 624 \\
\hline Serbia & Temporary & Monospecific & $43^{\circ} 29^{\prime} 24.2^{\prime \prime} \mathrm{N}$ & $19^{\circ} 52^{\prime} 06.9^{\prime \prime} \mathrm{E}$ & 949 & 9.6 & 839 \\
\hline Serbia & Temporary & Monospecific & $43^{\circ} 21^{\prime} 03.2^{\prime \prime} \mathrm{N}$ & $20^{\circ} 15^{\prime} 18.7^{\prime \prime} \mathrm{E}$ & 1470 & 6.7 & 821 \\
\hline Serbia & Temporary & Monospecific & $43^{\circ} 20^{\prime} 47.4^{\prime \prime} \mathrm{N}$ & $20^{\circ} 15^{\prime} 37.4^{\prime \prime} \mathrm{E}$ & 1650 & 5.7 & 819 \\
\hline Serbia & Temporary & Monospecific & $43^{\circ} 24^{\prime} 22.5^{\prime \prime} \mathrm{N}$ & $21^{\circ} 22^{\prime} 41.7^{\prime \prime} \mathrm{E}$ & 695 & 8.7 & 668 \\
\hline Slovakia & Temporary & Monospecific & $48^{\circ} 38^{\prime} 56.1^{\prime \prime} \mathrm{N}$ & $19^{\circ} 24^{\prime} 43.1^{\prime \prime} \mathrm{E}$ & 750 & 8.5 & 763 \\
\hline Slovakia & Temporary & Monospecific & $48^{\circ} 38^{\prime} 58.4^{\prime \prime} \mathrm{N}$ & $19^{\circ} 24^{\prime} 58.1^{\prime \prime} \mathrm{E}$ & 750 & 8.5 & 763 \\
\hline Slovenia & Temporary & Monospecific & $46^{\circ} 06^{\prime} 56.1^{\prime \prime} \mathrm{N}$ & $15^{\circ} 03^{\prime} 42.8^{\prime \prime} \mathrm{E}$ & 600 & 10.6 & 1223 \\
\hline Slovenia & Temporary & Monospecific & $46^{\circ} 05^{\prime} 38.3^{\prime \prime} \mathrm{N}$ & $15^{\circ} 03^{\prime} 58.7^{\prime \prime} \mathrm{E}$ & 1070 & 8.2 & 1223 \\
\hline Spain & Temporary & Monospecific & $42^{\circ} 16^{\prime} 23.6^{\prime \prime} \mathrm{N}$ & $3^{\circ} 16^{\prime} 12.6^{\prime \prime} \mathrm{W}$ & 1526 & 9.5 & 631 \\
\hline Spain & Temporary & Monospecific & $43^{\circ} 07^{\prime} 40^{\prime \prime} \mathrm{N}$ & $4^{\circ} 58^{\prime} 30^{\prime \prime} \mathrm{W}$ & 1279 & 10.0 & 900 \\
\hline Spain & Temporary & Monospecific & $43^{\circ} 07^{\prime} 38^{\prime \prime} \mathrm{N}$ & $4^{\circ} 58^{\prime} 48^{\prime \prime} \mathrm{W}$ & 1474 & 9.0 & 900 \\
\hline Spain & Temporary & Monospecific & $42^{\circ} 12^{\prime} 05^{\prime \prime} \mathrm{N}$ & $2^{\circ} 43^{\prime} 19^{\prime \prime} \mathrm{W}$ & 1430 & 10.5 & 575 \\
\hline Spain & Temporary & Monospecific & $42^{\circ} 12^{\prime} 06^{\prime \prime} \mathrm{N}$ & $2^{\circ} 43^{\prime} 34^{\prime \prime} \mathrm{W}$ & 1430 & 10.5 & 575 \\
\hline Spain & Temporary & Monospecific & $41^{\circ} 46^{\prime} 32^{\prime \prime} \mathrm{N}$ & $2^{\circ} 27^{\prime} 24^{\prime \prime} \mathrm{E}$ & 1186 & 11.6 & 711 \\
\hline Spain & Temporary & Monospecific & $41^{\circ} 46^{\prime} 30^{\prime \prime} \mathrm{N}$ & $2^{\circ} 27^{\prime} 26^{\prime \prime} \mathrm{E}$ & 1183 & 11.7 & 711 \\
\hline Ukraine & Temporary & Monospecific & $49^{\circ} 02^{\prime} 01^{\prime \prime} \mathrm{N}$ & $23^{\circ} 33^{\prime} 11^{\prime \prime} \mathrm{E}$ & 586 & 8.8 & 946 \\
\hline Ukraine & Temporary & Monospecific & $49^{\circ} 02^{\prime} 01^{\prime \prime} \mathrm{N}$ & $23^{\circ} 33^{\prime} 16^{\prime \prime} \mathrm{E}$ & 578 & 8.9 & 946 \\
\hline Ukraine & Temporary & Monospecific & $49^{\circ} 03^{\prime} 48^{\prime \prime} \mathrm{N}$ & $22^{\circ} 53^{\prime} 44^{\prime \prime} \mathrm{E}$ & 892 & 7.2 & 1000 \\
\hline Ukraine & Temporary & Monospecific & $49^{\circ} 03^{\prime} 41^{\prime \prime} \mathrm{N}$ & $22^{\circ} 53^{\prime} 15^{\prime \prime} \mathrm{E}$ & 908 & 7.2 & 1000 \\
\hline
\end{tabular}

$E$ elevation (m a.s.1.), $T$ mean annual temperature $\left({ }^{\circ} \mathrm{C}\right), P$ total annual precipitation (mm). Climate data is shown as mean of the period 1971-2000 (CRU database; Harris et al. 2020)

from around $600 \mathrm{~m}$ a.s.l. to more than $1600 \mathrm{~m}$ a.s.l., both in monospecific and mixed stands. The sites cover a large range of climate conditions with mean temperatures between 2.6 and $10.2{ }^{\circ} \mathrm{C}$ and annual precipitation between 517 and $2780 \mathrm{~mm}$ (Fig. 5.4).

\subsection{Stand Growth}

The 159 observational plots included in the network are located in such a way that they represent a broad environmental gradient (Figs. 5.5 and 5.6, Table 5.4). Table 5.5 gives an overview of the characteristics of the forest stands, which are included in the network. Even though the observation period of the temporary 


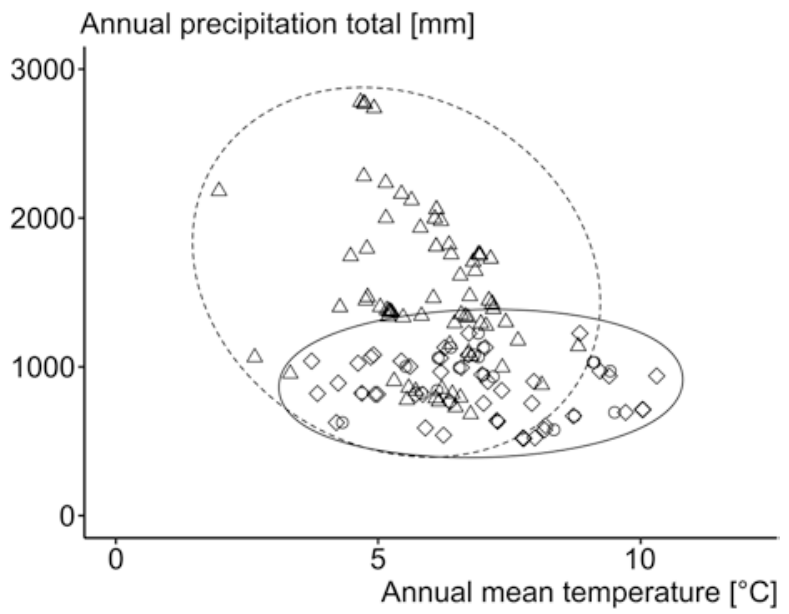

Fig. 5.4 Scatter plot of the total annual precipitation in mm and mean annual temperature in ${ }^{\circ} \mathrm{C}$ of the 89 long-term observational plots in mixed mountain forests (triangles) and 72 temporary observational plots in monospecific stands of beech (rhombuses) and mixed mountain forest plots (circles). Ellipses represent a convex hull of the temporary plots (solid line) and long-term observational plots in mixed mountain forests (dashed)

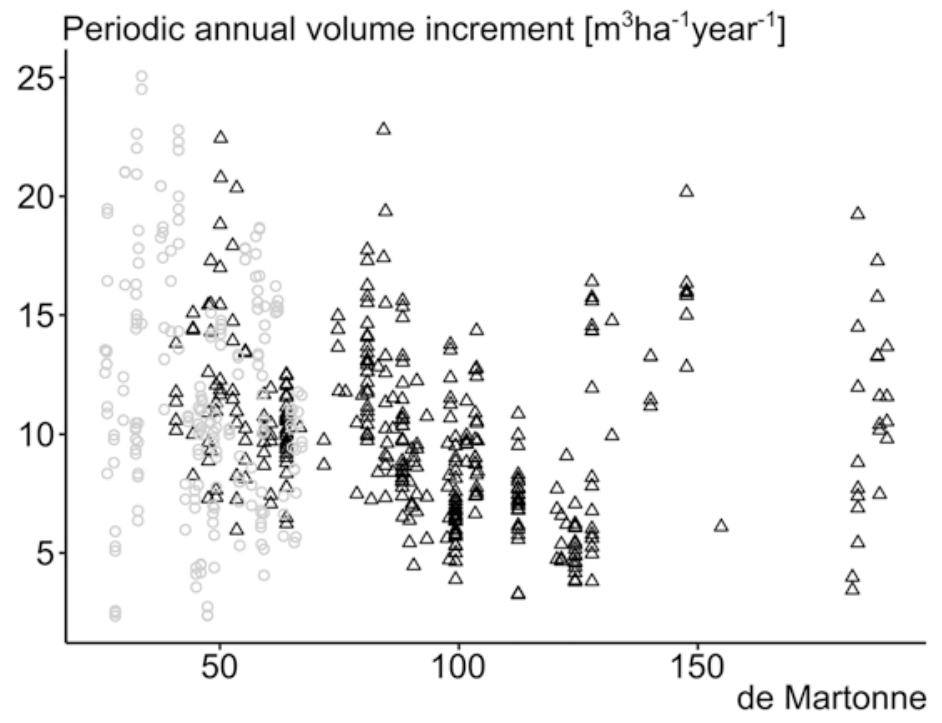

Fig. 5.5 Stand periodic annual volume increment, $\mathrm{m}^{3} \mathrm{ha}^{-1}$ year ${ }^{-1}$ over de Martonne aridity index, $\mathrm{mm}{ }^{\circ} \mathrm{C}^{-1}$ (de Martonne 1926). Long-term observational plots are shown with black triangles; temporary observational plots are shown with grey circles. Note that the values for periodic annual volume increments on the temporary plots were derived retrospectively (Heym et al. 2017, 2018) 


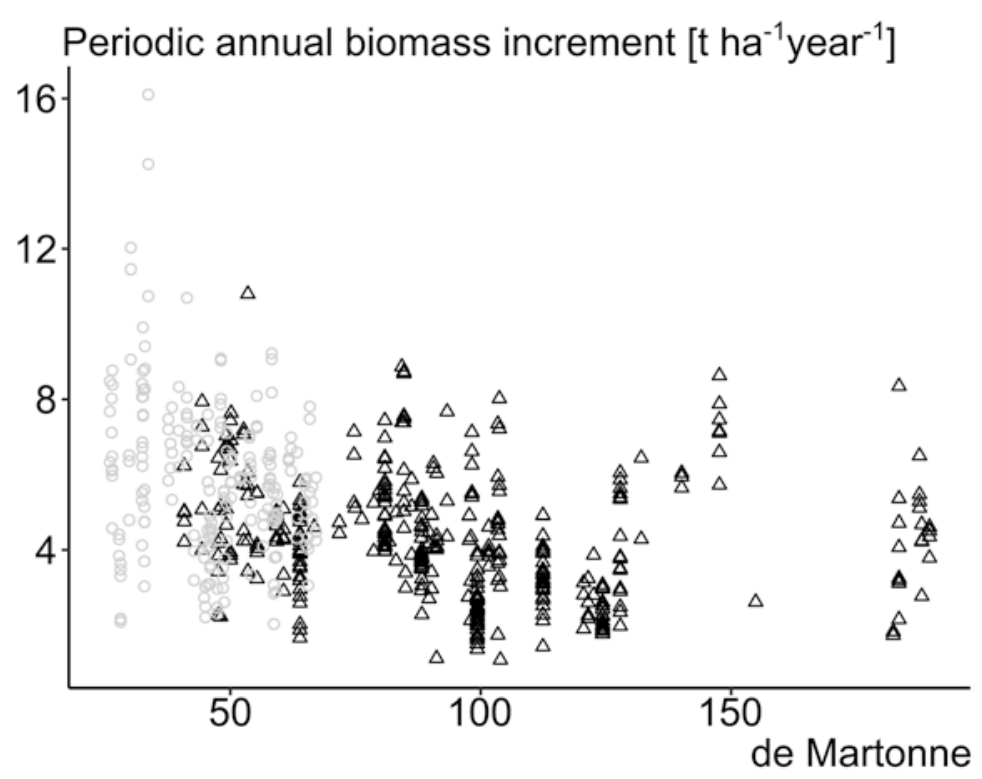

Fig. 5.6 Stand periodic annual biomass increment, $\mathrm{t} \mathrm{ha}^{-1}$ year $^{-1}$ over de Martonne aridity index, $\mathrm{mm}^{\circ} \mathrm{C}^{-1}$ (de Martonne 1926). Long-term observational plots are shown with black triangles; temporary observational plots are shown with grey circles. Biomass was calculated by generalized biomass allometric equations (Forrester et al. 2017). Note that the values for periodic annual biomass increments on the temporary plots were derived retrospectively (Heym et al. 2017, 2018)

observational plots by retrospective calculation (see Sect. 5.3.1) only dates back to the year 2007, the data of the long-term experimental plots in particular offer a broad data basis for investigating productivity in terms of merchantable volume over bark and biomass of mountain forests at elevations of 600-1600 m a.s.l. in Europe (Fig. 5.2). The observation horizon of the long-term experimental plots reaches back several decades in most cases (Fig. 5.7) and, therefore, the dataset could be also used to answer the question of whether stand growth has changed in recent decades and how these changes are related to changes in climate conditions or forest structure (Torresan et al. 2020). The dataset is also suitable for testing if there was a shift in species-specific productivity of European beech, Norway spruce, or silver fir over recent decades and whether this shift was influenced by the mixture (Hilmers et al. 2019). Furthermore, the data could be used to answer questions, such as how productivity depends on site conditions and how these vary across geographical regions (Figs. 5.5 and 5.6). They also permit quantifying tree neighbourhood effects and analysing how climate affects the mechanisms of forest dynamics as well as inferring cause-effect relationships between disturbance events (e.g. droughts, outbreaks) and demographic changes (i.e. mortality and recruitment) (Lutz 2015). 
至

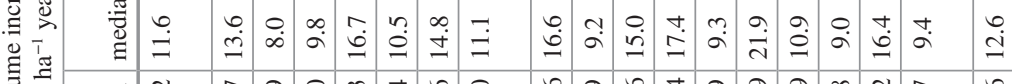

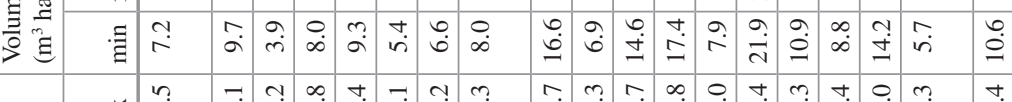

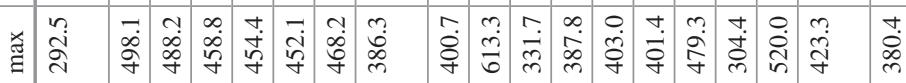

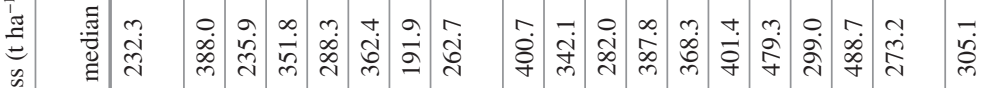

晃

$\stackrel{\mathscr{J}}{\overparen{a}}$

突

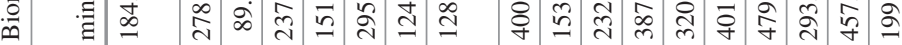

호

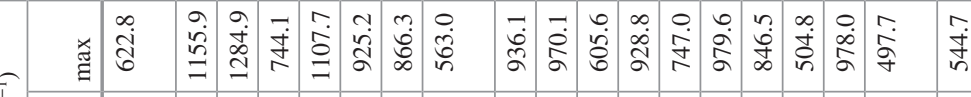

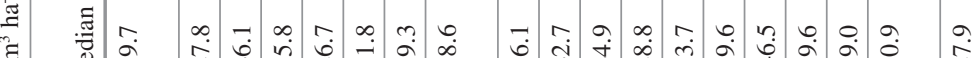

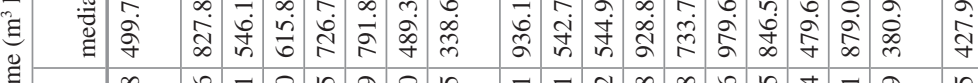

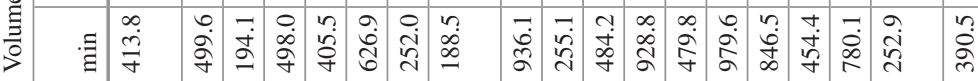

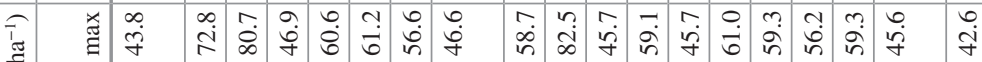

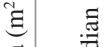

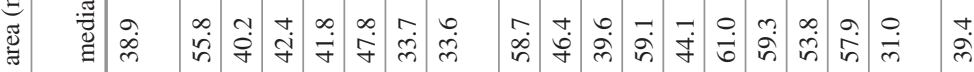

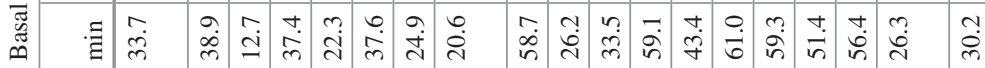

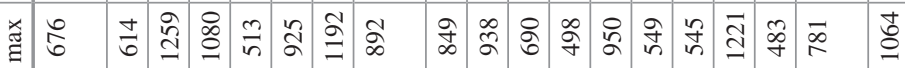

음

$\pm$

4

.

.



苞

$\Xi$

3

$\sum_{\substack{0 \\ 0}}^{\frac{0}{2}}$

₹

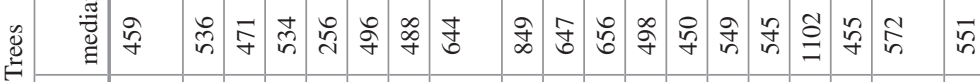

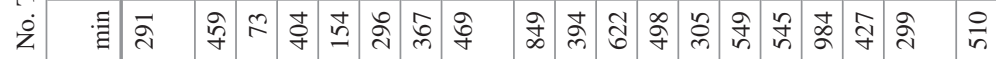

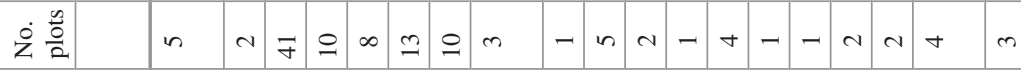

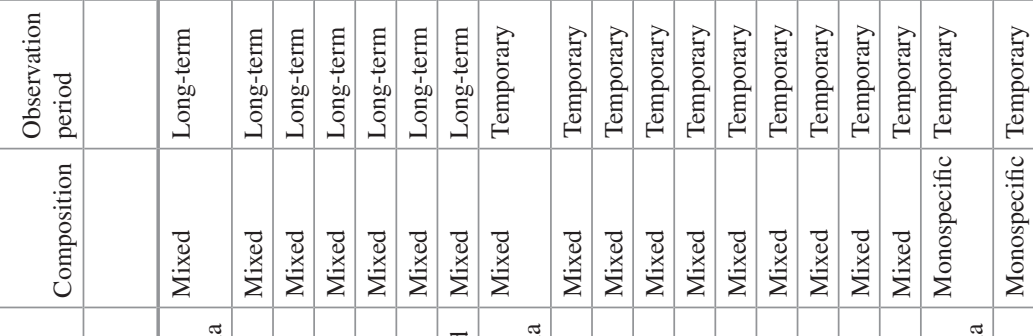

in

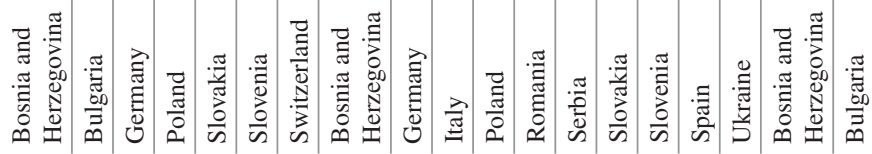




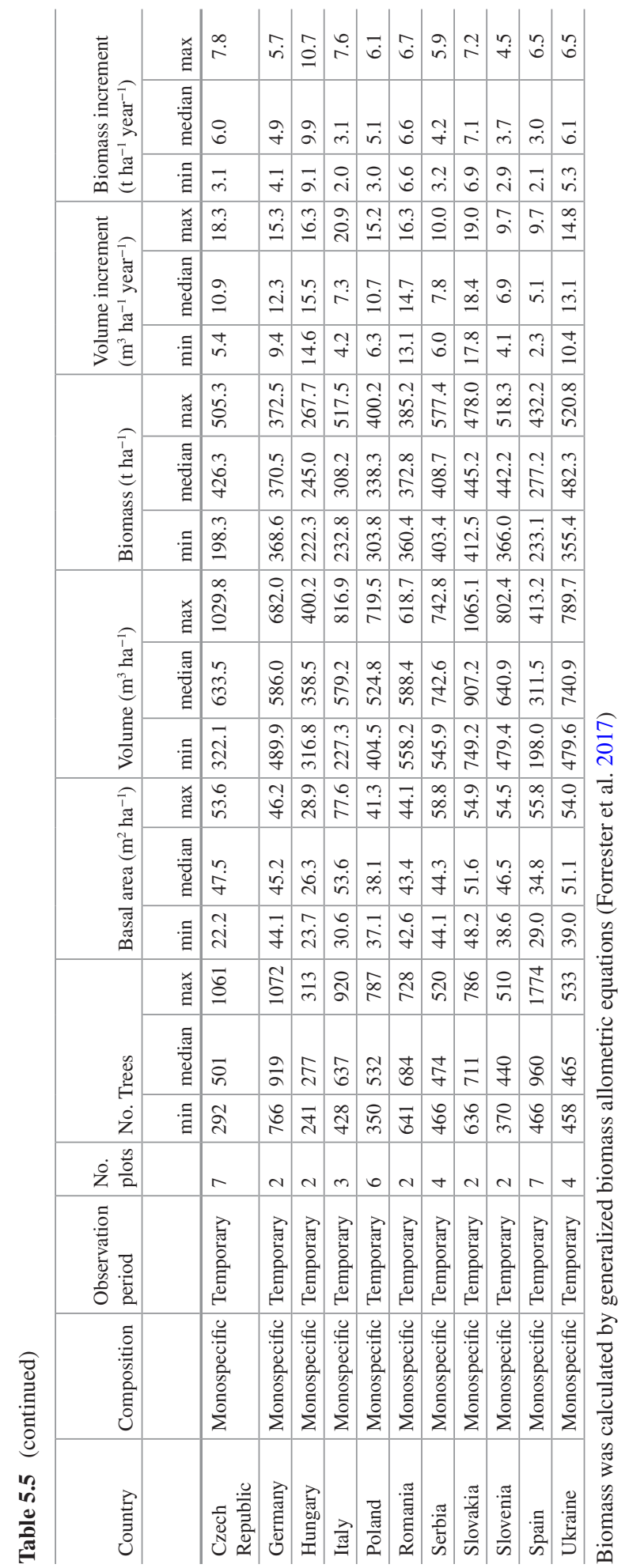




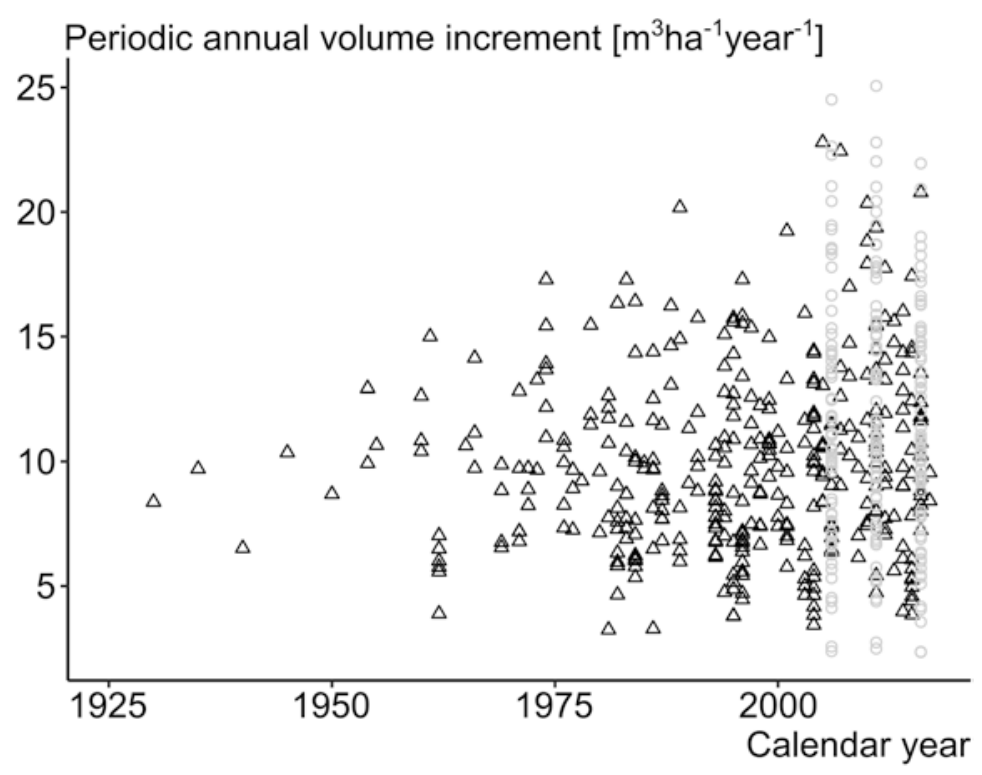

Fig. 5.7 Stand periodic annual volume increment, $\mathrm{m}^{3} \mathrm{ha}^{-1}$ year ${ }^{-1}$ over the calendar years. Longterm observational plots are shown with black triangles; temporary observational plots are shown with grey circles. Note that the values for periodic annual volume increments on the temporary plots were derived retrospectively (Heym et al. 2017, 2018)

\subsection{Tree Growth}

From trees on the temporary plots and from trees in the buffer zone around the longterm plots, cores of 2437 European beech, 479 Norway spruce, and 907 silver fir trees were collected. Table 5.6 provides an overview of the tree-ring data. Some of the trees were up to 600 years old at the time of data collection, so that growth over several centuries could be analysed (Fig. 5.8, Table 5.6). With this dataset, questions can be answered as to whether the growth of the trees has changed over the long term and if there were species-specific changes in the growth trends during the last centuries (Figs. 5.8 and 5.9; Pretzsch et al. 2020a, b). Questions can also be answered at the single-tree level as to how tree growth changed along the site gradient and if there were different tree-growth patterns in mixed compared to monospecific stands (see Chap. 6 of this book: Pretzsch et al. 2021). Insights on radial growth responses to climate dynamics and extreme events in mixed versus pure stands of these species can also be gathered at specific sites of the network (e.g. Versace et al. 2020), as well as address questions on competitive interactions (e.g. Versace et al. 2019). Tree-growth series from mixed mountain forests can be also used to explore spatialtemporal patterns of the intra- and interspecific growth synchrony related to climate variation during the past century, which can help to identify between species temporal complementarity and the role of mixed mountain forests in the frame of climate change (del Río et al. 2021). 


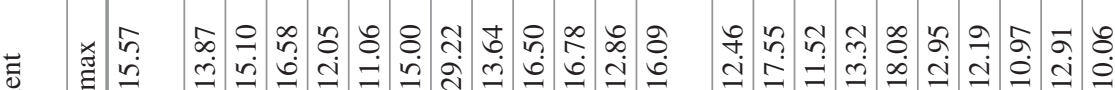
v

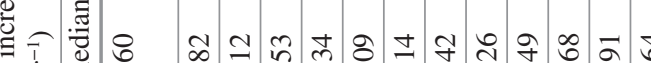

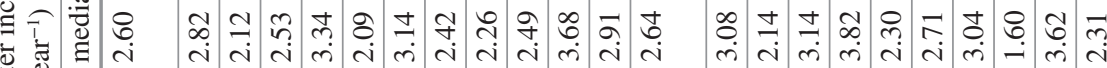
(2) 䨔

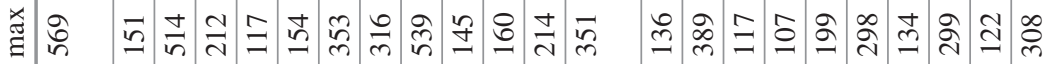
จิ $:$ :

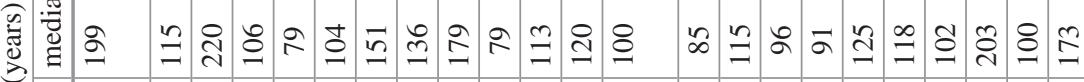

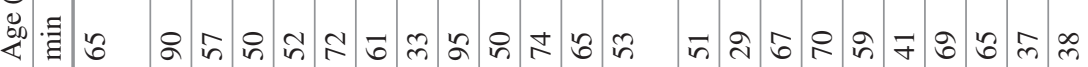

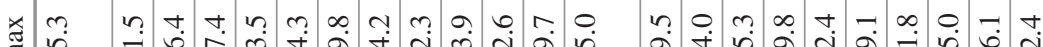

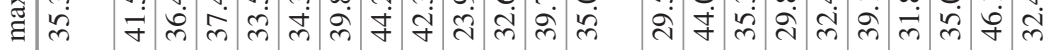
สี

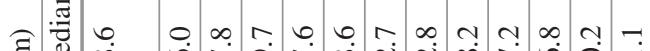
छ

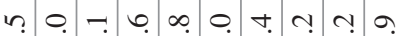
.0.00

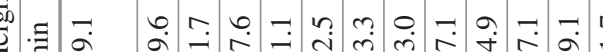

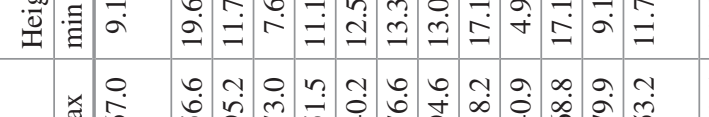

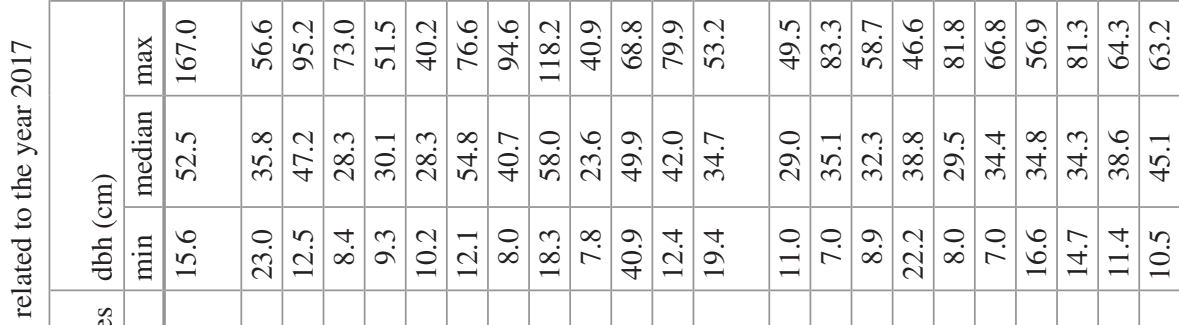
宖芜

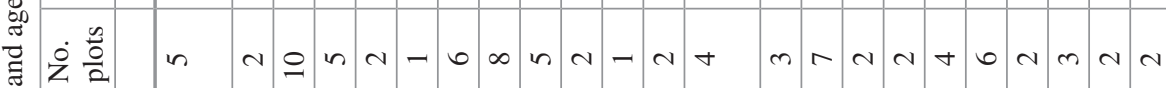

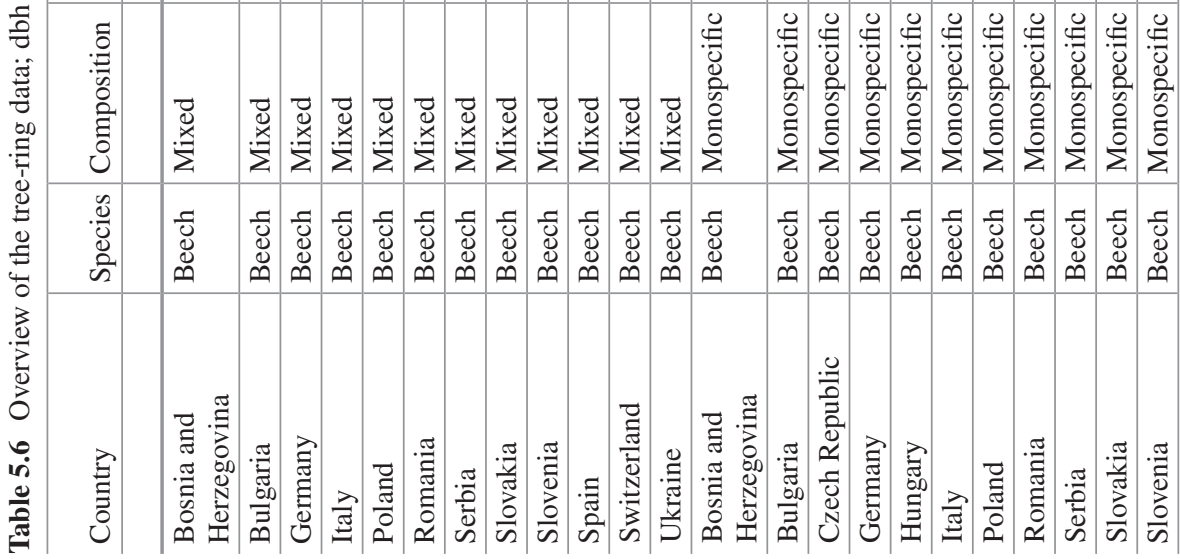


5 Efficacy of Trans-geographic Observational Network Design for Revelation...

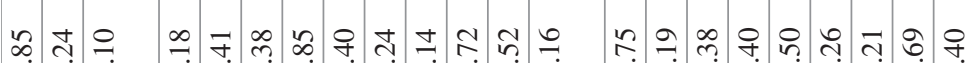

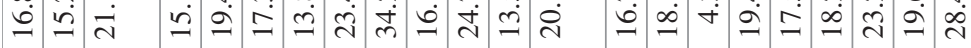

๓

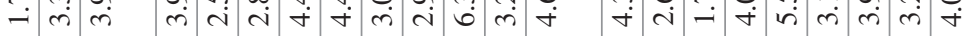
官

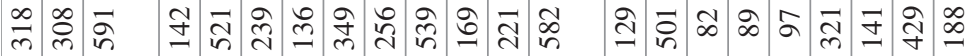

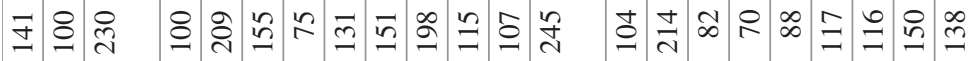

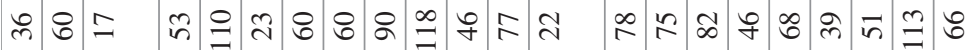

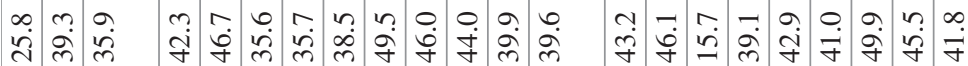
t. I ते

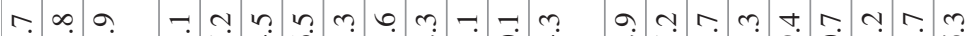

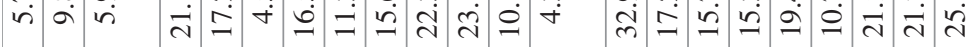
๖.

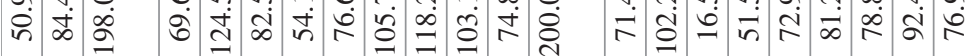

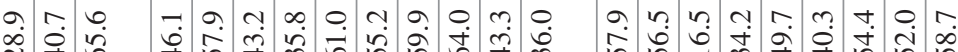

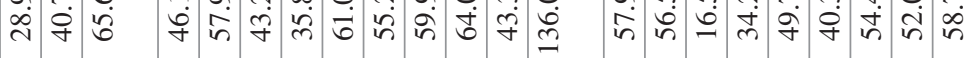
$0.0 \%$ ․

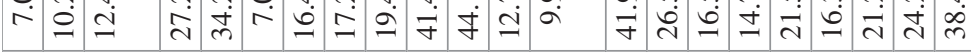

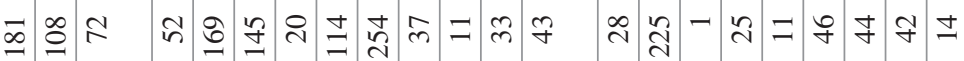

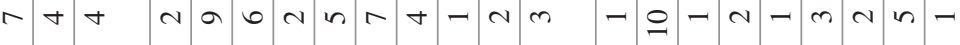
$\because \stackrel{\bullet}{=}$

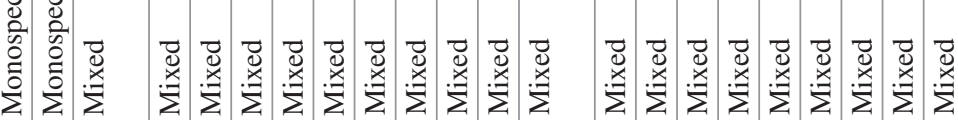

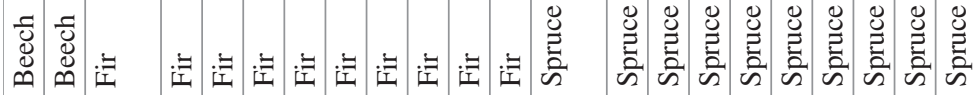

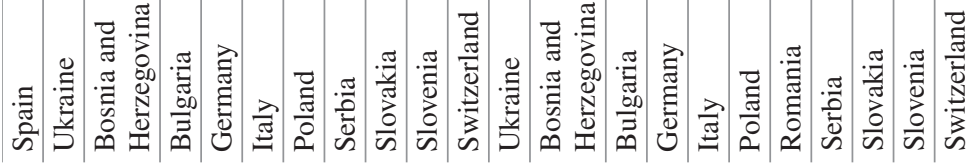



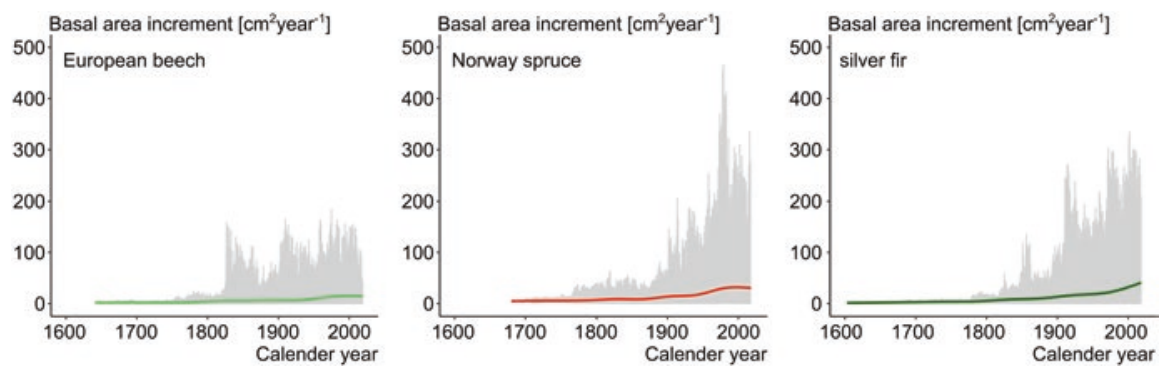

Fig. 5.8 Single-tree basal increment in $\mathrm{cm}^{2}$ year ${ }^{-1}$ over the calendar years of European beech, Norway spruce, and silver fir in the long-term and temporary observational forest plots. Coloured lines were generated by fitting a loess curve
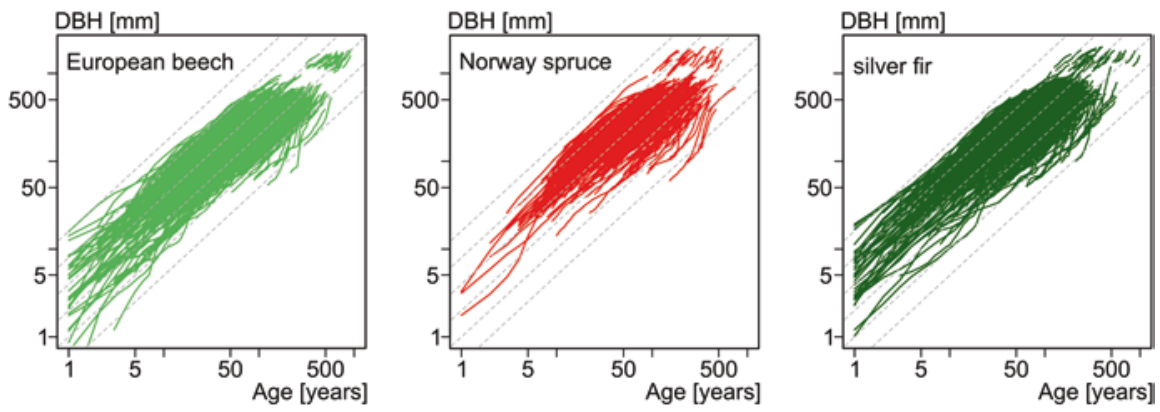

Fig. 5.9 Stem diameter-tree age trajectories of the 2437 European beech, 479 Norway spruce, and 907 silver fir trees during the last few centuries, in double logarithmic representation. Most trees show a linear increase in stem diameter with progressing age (reference lines $\ln \ln (d)=a_{0}+a_{1} \ln \ln ($ age $)$ with $a_{0}=1$ and varying $a_{0}$ ) and no asymptotic growth curve pattern

\subsection{Growth Characteristics Analysed Along Elevation Gradients}

The data collected from the observational plots cover a wide range along an elevation gradient of $\sim 600-1600 \mathrm{~m}$ a.s.1. (Figs. 5.10, 5.11 and 5.12). Both at stand level (Fig. 5.10; Hilmers et al. 2019) and at single-tree level (Fig. 5.11; Pretzsch et al. 2020a, b), it can be investigated if there are any growth trends along the elevation gradient and whether these trends have changed during the last century. Furthermore, the dataset offers the possibility to analyse if the elevation-dependent growth trends were different in the mixed compared to the monospecific stands and how the mixture, i.e. the interspecific competition, influences such changes (Figs. 5.11 and 5.12). Accordingly, intra- and interspecific growth synchrony in response to interannual fluctuations, as an indicator of tree species dependence on climate variability, can vary along the elevation gradient (del Río et al. 2021). 


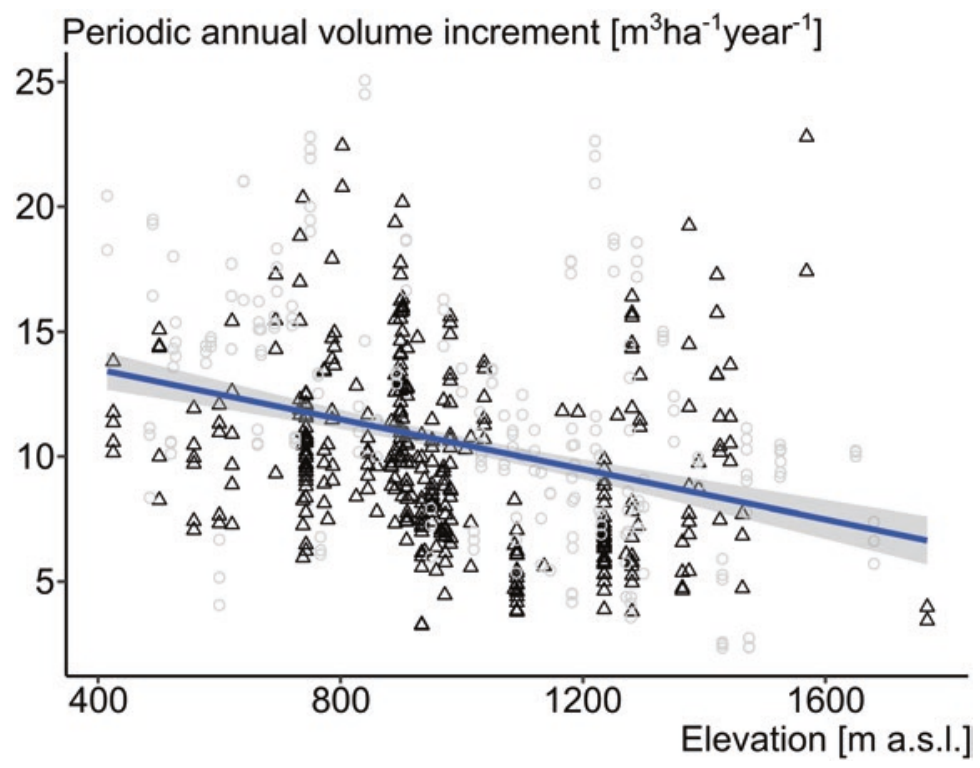

Fig. 5.10 Stand periodic annual volume increment (PAIV) in $\mathrm{m}^{3} \mathrm{ha}^{-1}$ year $^{-1}$ over elevation in $\mathrm{m}$ a.s.l. Black triangles represent long-term observational mixed mountain forests; grey circles show the PAIV of the temporary observational plots in monospecific beech stands and mixed mountain forests. The blue line is based on a linear model and the grey area indicates the $95 \%$ confidence interval. Note that the values for PAIV on the temporary plots were derived retrospectively (Heym et al. 2017, 2018)
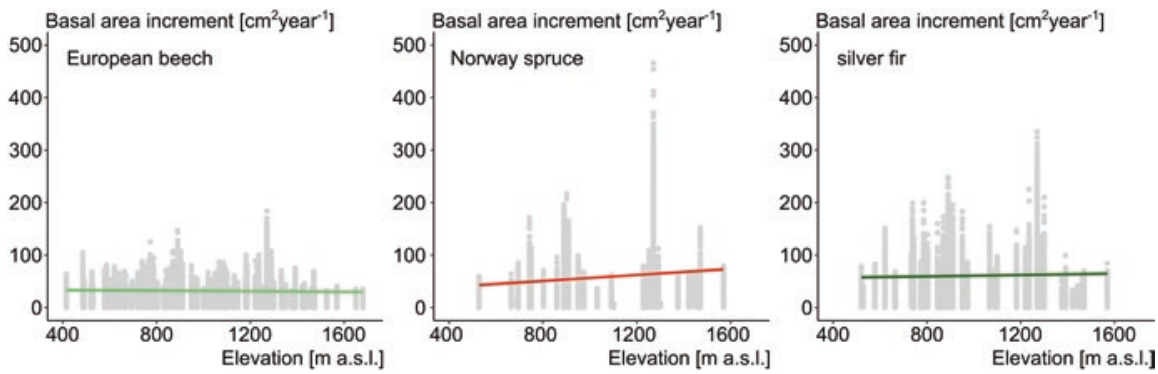

Fig. 5.11 Single-tree basal area increment in $\mathrm{cm}^{2}$ year ${ }^{-1}$ over elevation in $\mathrm{m}$ a.s.l. of European beech, Norway spruce, and silver fir in the long-term and temporary observational forest plots. Coloured lines are based on linear models 

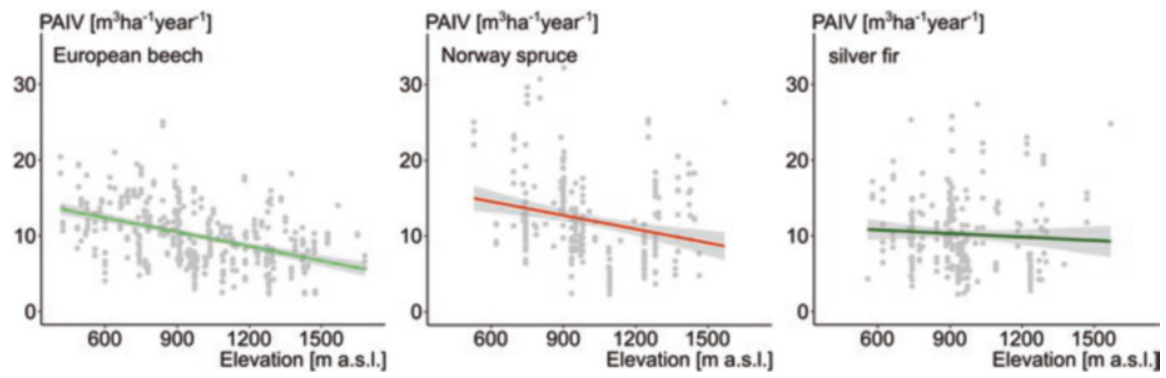

Fig. 5.12 Stand periodic annual volume increment (PAIV) in $\mathrm{m}^{3} \mathrm{ha}^{-1}$ year ${ }^{-1}$ over elevation in $\mathrm{m}$ a.s.l of European beech, Norway spruce, and silver fir in the long-term and temporary observational forest plots. The periodic annual volume increment of the three tree species was scaled using the species share derived from SDI proportions (Pretzsch and Biber 2016). The coloured lines are based on linear models and the grey areas indicate the 95\% confidence interval. Note that the values for PAIV on the temporary plots were derived retrospectively (Heym et al. 2017, 2018)

\subsection{Concept of Statistical Evaluation of Drought Events}

The analysis of tree growth-related data (e.g. tree-ring width, basal area increment, or stable isotope signatures) with the aim of detecting significant event years related to critical conditions for growth, such as droughts, firstly requires the use of reliable climate indices allowing their identification. Simpler indices can be applied, such as the de Martonne index (de Martonne 1926), which only requires temperature and precipitation data. In addition, more developed indices including also potential evapotranspiration, such as the PDSI (Palmer Drought Severity Index) (Palmer 1965) or the SPEI (Standardized Precipitation-Evapotranspiration Index) (VicenteSerrano et al. 2010), have been developed to carefully select those years that stand out both in the short term (few years) and in the long term from the average climate trend of a specific region.

To infer the response of trees to drought events, an increasingly adopted approach is that of the Lloret indices (Lloret et al. 2011). They allow estimating, for each considered year in a tree-ring series, three components explaining the growth during and after the event itself. These are the resistance $(R t)$, recovery $(R c)$, and resilience $(R s)$, i.e. the ratio between growth during and before the drought event, after and during the drought event, and after and before the drought event, respectively. Conceptually, resistance represents the ability of a tree to cope with the stressful event during the same growing season, by possibly maintaining the previous growth rates, and hence is the most significant parameter to evaluate. Resilience and recovery are, on the other hand, related to the ability of the tree to sustain precedent growth rates in the short term after the event. It is, therefore, necessary to carefully select the time frame, in terms of years, of the average pre- and post-drought growth rate and to adjust it to species-specific requirements related to ecophysiology. The 
selection of the samples to be included in the resilience analysis is also of paramount importance. For instance, depending on the position of the tree crown within the vertical layering of the stand, the growth response to a drought event might differ consistently. Thus, separating dominant from dominated individuals and proceeding to differential modelling of their growth patterns are crucial to obtain consistent results. Recently, the Lloret indices have been integrated in an R package, called "pointRes" (Van der Maaten-Theunissen et al. 2015), which allows detecting both negative and positive pointer years, information that can be combined with that of drought indices to improve the accuracy of the investigation and to allow evaluating the growth response also during particularly favourable growing conditions. Normally, the resistance, resilience, and recovery analyses can be carried out on specific, pre-selected event years. It might be of interest, however, also to apply the above-mentioned Lloret indices to continuous, long-term series in order to investigate the response of trees to increasingly frequent drought events, which are affecting not only the growth patterns in the short term but also in the mid- and likely in the long term. In this context, it becomes relevant the removal of autocorrelation from the tree-growth series, using for instance ARMA (autoregressive moving average) or ARIMA (autoregressive integrated moving average) models and calculating $R t, R c$, and $R s$ on the residuals (Fig. 5.13).

When studying forests' response to drought events, it is possible to work at different spatial scales, i.e. tree level and stand level. Therefore, it is fundamental to consider the hierarchical and non-independent structure of the data and to assess possible nesting effects. To this extent, mixed models with random and fixed effects proved to be a valid solution, as they allow considering the above-mentioned issues, e.g. separating site- and plot-related variability from that linked to the factors of interest, such as species mixing and forest vertical and horizontal structure.

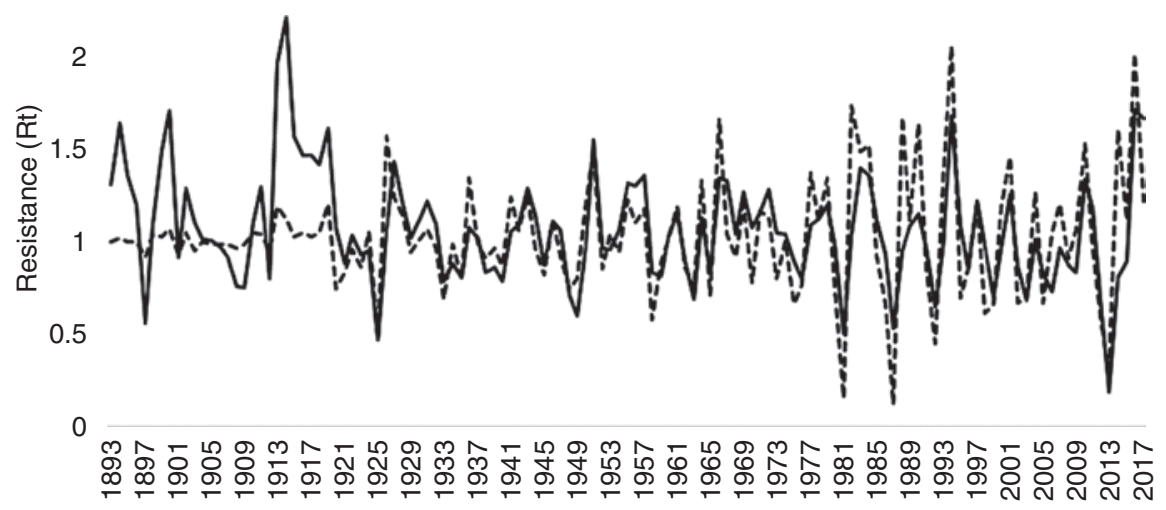

Fig. 5.13 Resistance index (Rt) calculated on a sample beech tree. The bold line represents the $R t$ calculated on basal area increment (BAI) directly, whereas the dashed line is the same index calculated on the BAI residuals after ARMA modelling 


\subsection{Climate Smartness}

\subsubsection{Assessing Climate-Smart Indicators}

In order to assess climate smartness of forestry on stand level, experimental plots were evaluated on the basis of selected indicators. These were based on the Forest Europe C\&I (Forest Europe 2015) set for sustainable forest management (https:// foresteurope.org/sfm-criteria-indicators/). The assessment included a subset of 17 indicators, which were recorded on monospecific beech as well as mixed beech, spruce, and fir stands (Table 5.7). The selected indicators are characterized in particular by an area reference and a simple assessment method, which can be standardized for any region. Each indicator is characterized by measurable characteristics of assessment, which allow a more detailed evaluation (Table 5.7; see Chap. 6 of this book: Pretzsch et al. 2021). The assessment follows the procedure developed by Pfatrisch (2019).

In a recent exercise on literature review and multicriteria analysis, Santopuoli et al. (2020) highlighted that a subset of 10 indicators, from the current PanEuropean C\&I set (Forest Europe 2015), were more frequently used to assess adaptation and mitigation management strategies of forest ecosystems. In particular, carbon stock (mitigation), tree species composition (adaptation and mitigation), and forest damage (adaptation and mitigation) showed the highest priority value.

Table 5.7 Selected climate-smart indicators for field assessment according to the method of Pfatrisch (2019)

\begin{tabular}{|c|c|c|}
\hline $\mathrm{Nr}$ & Indicator & Characteristic of assessment $(n)$ \\
\hline 1.2 & Growing stock & Growing stock (1) \\
\hline 1.3 & Diameter distribution & Diameter distribution (1) \\
\hline 1.4 & Carbon stock & Carbon Stock, development, substitution (3) \\
\hline 2.3 & Defoliation & Defoliation (1) \\
\hline 2.4 & Forest damage & Risk, impact, number of damages (3) \\
\hline 2.5 & Stability & Slenderness coefficient, tree height, stand density (3) \\
\hline 3.1 & Increment and felling & Increment, felling, relation (3) \\
\hline 4.1 & Tree species composition & Tree species composition (1) \\
\hline 4.2 & Regeneration & Area, height, density, potential, browsing (5) \\
\hline 4.3 & Naturalness & Naturalness (sp. comp.), soil scarification (2) \\
\hline 4.4 & Introduced tree species & Introduced tree species (1) \\
\hline 4.5 & Deadwood & $\begin{array}{l}\text { Quantity, standing volume, decomposition rate, light } \\
\text { exposure, stand age (5) }\end{array}$ \\
\hline 4.6 & Genetic resources & Phenotypic similarity, gen conservation (2) \\
\hline 4.8 & $\begin{array}{l}\text { Threatened forest } \\
\text { species }\end{array}$ & Threatened forest species (1) \\
\hline $4.91 / 2$ & $\begin{array}{l}\text { Distribution of tree } \\
\text { crowns }\end{array}$ & Canopy level (horizontal), crown layers (vertical) (2) \\
\hline 6.1 & $\begin{array}{l}\text { Accessibility (for } \\
\text { recreation) }\end{array}$ & Distance to road, road density (2) \\
\hline
\end{tabular}

Each indicator had 1-5 characteristics of assessment 


\subsubsection{European Dataset of Climate-Smart Indicators}

The assessment of the climate-smart indicators was carried out on 46 trial plots in six different countries. The experimental plots cover a large elevation gradient from 40 to $1463 \mathrm{~m}$ above sea level as well as broad spatial gradients and different management types (Table 5.8). The resulting European-wide dataset allows an analysis of climate smartness at European and regional level with country- and stand-specific properties. In Chap. 6 of this book (Pretzsch et al. 2021), a more detailed information about climate smartness assessment is presented using spruce-fir-beech mixed mountain forests as case study.

\subsubsection{Linking Yield and Climate-Smart Indicators: Research Objectives}

The presented approach of climate smartness assessment is a promising way to analyse and estimate substantive yield data concerning climate smartness in forest management. With the evaluated effects, an optimized list of indicators can contribute to a more effective and sustainable climate-smart forestry. An iterative participatory process with input from experts (CLIMO) has recently provided the basis for developing a comprehensive and shared definition of climate-smart forestry (Bowditch et al. 2020). On the European level, climate-smart forestry indicators supported by network analysis approach can help to deepen the understanding about the development of mountain mixed forests along a broad geographical gradient. Linking yield data from long-term experimental plots with climate-smart indicators can provide valuable insights in forest dynamics with special regard to climate smartness. The obtained results can provide the basis for improvement of a futureoriented European forest management as well as regional silvicultural guidelines.

\subsection{Soils}

Soils in native forests rarely experience significant disturbances that are more common for soils in other land-use systems. However, climate change and/or deforestation can cause dramatic changes in the quality of forest soils. Alteration of quality or quantity of soil organic matter in forest soils is one of the most important consequences of climate change (Raison and Khanna 2011). Deforestation causes change in hydrological processes, which can enhance surface runoff and soil erosion, increase the recharge of groundwater and cause the reduction of organic carbon, nitrogen, phosphorus, and exchangeable potassium, calcium, and magnesium (Pennock and van Kessel 1997). The rate of soil degradation in such changed conditions depends largely on the type of bedrock, which was until recently considered of subordinate significance compared to climate and pedological characteristics (Jiang et al. 2020). Not only bedrock has a significant role in vegetation growth by 


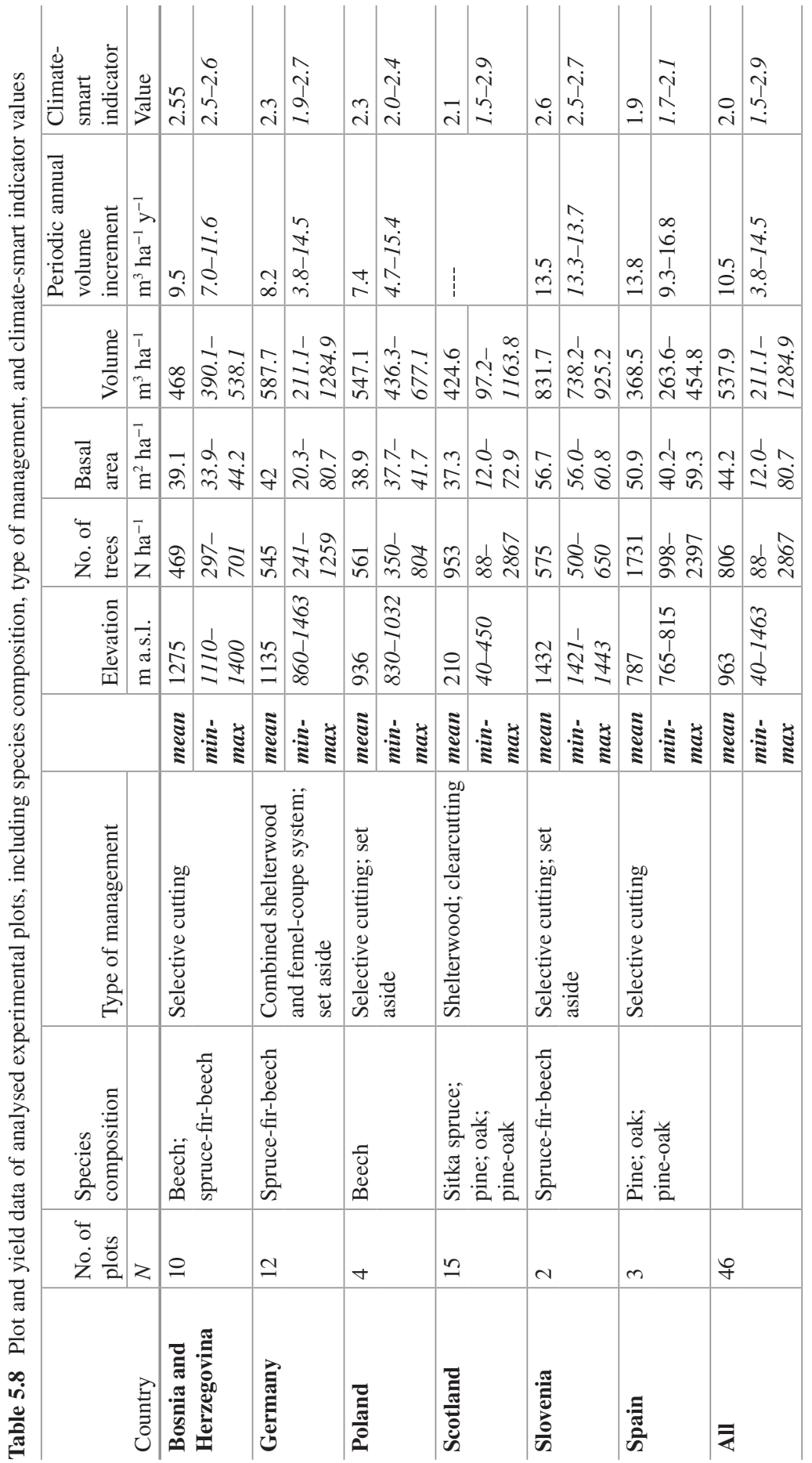


regulating physical and chemical properties in soils, but it can also change the response of vegetation to climate factors (Jiang et al. 2020).

The multilayer and comprehensive network of beech in European mountains (study 2; see Sect. 5.4) contains soil data that can be used for spatial and temporal analyses of soil quality, testing existing and establishing new erosion indices, and predicting landform processes in the case of climate and land-use changes. This dataset contains a total of 76 soil samples from 20 pure beech forest stands from Bosnia and Herzegovina, Bulgaria, Czech Republic, Germany, Italy, Poland, Romania, Serbia, Slovakia, Slovenia, and Spain. Five types of bedrock were identified: limestone ( 8 plots, 28 samples), sandstone (5 plots, 20 samples), granite (5 plots, 20 samples), quartzite (1 plot, 4 samples), and andesite (1 plot, 4 samples). The Manual for Sampling and Analysis of Soils (Carter and Gregorich 2007) was followed for soil sampling and analyses. Soil samples were collected at 4 depths along the soil profiles $(0-10,10-20,20-40$, and $40-80 \mathrm{~cm})$. The following soil characteristics were determined on all samples: $\mathrm{pH}$; electrical conductivity; redox potential; content of $\mathrm{CaCO}_{3}$; content of aggregates $>2 \mathrm{~mm}$; grain size composition; content of organic carbon and nitrogen; concentration of available ions $\mathrm{Al}, \mathrm{Ca}, \mathrm{Mg}$, $\mathrm{K}, \mathrm{Na}, \mathrm{Mn}$, and $\mathrm{Fe}$; content of major and minor elements; and a concentration of carbonate, nitrite, sulphate, nitrate, bromide, oxalate, phosphate, fluoride, and chloride ions.

One of the most apparent results is that bedrock properties play a significant role in beech forest soil characteristics (Fig. 5.14). The PCA separated stands on limestone with the highest content of organic carbon (average 4.51\%), $\mathrm{pH}$ of 6.2, and high content of exchangeable $\mathrm{Ca}, \mathrm{Mg}$, and $\mathrm{K}$ (24.04 ppm, $9.24 \mathrm{ppm}$, and $13.35 \mathrm{ppm}$, respectively) from sandstone and granite substrates. Soils on sandstone have 4 times and granite soils have 3.6 times lower content of organic carbon. Soil pH for both sandstone and granite bedrock is 4.9 , and the exchangeable $\mathrm{K}$ is 0.6 times lower than limestone soils. Quartzite and andesite soils have similar characteristics to granite soils but cannot be further discussed due to the small number of samples. A group of soils with the bedrock that is classified as limestone are not typical carbonate rocks but are limestone moraine and calcareous sandstone, and although they
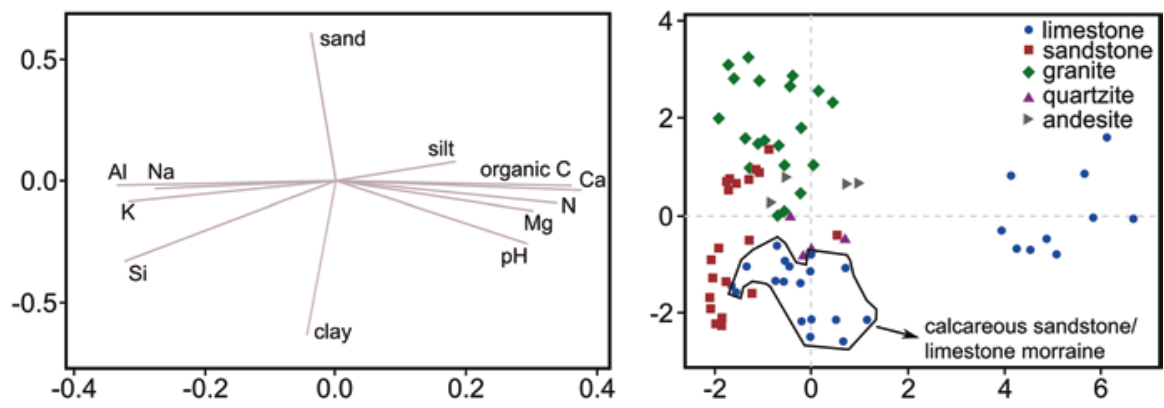

Fig. 5.14 Principal component analyses of pure beech forest soils developed on a range of bedrock types 
have some characteristics of limestone soils, they are also by their textural and mineralogical properties close to sandstones (Fig. 5.14).

Obtained results are a good indication that soils on different bedrocks respond differently to environmental changes. Further investigations are aimed towards exploring existing soil erosion indices and developing new indicators that would be more appropriate for forest soils. Such information would be of great assistance for forest management and possible mitigation measures against climate change.

\subsection{Genetic Resources}

Evaluating genetic structure of forest stands provides insights into in situ surviving potential of tree species and their population. High genetic variation and elevated diversity of alleles assume long-term persistence, providing a good chance for populations to adapt to changing environments (Falk et al. 2006). In the past three decades, molecular techniques have become more and more available to determine the quality and quantity of the genetic diversity preserved within and among populations. Studies have shown the overall high genetic potential in forest tree populations as most of the species exhibited high within-population genetic diversity and relatively low divergence among adjacent populations (Porth and El-Kassaby 2014). Moreover, genetic variation is associated with the geographic distribution of the alleles and depends on the past historical events and demographic processes of populations. Accordingly, the levels of population genetic variation exhibit spatial and temporal differences that should be taken into consideration in any evaluation, like nature conservation activity or when forest management is designed (van Dam 2002).

Mountain forests are among the most exposed ecosystems to climate change (Becker and Bugmann 2001; Nogués-Bravo et al. 2007). Due to the fast environmental shifts, selective pressure acts on the genetic constitution of mountain tree species. European beech, as one of the most widespread trees of the European mountain forests, is presumed to be heavily affected (Kramer et al. 2010). However, beech has been thoroughly investigated because of its wide distribution and due to the great economic importance, so that a wealth of ecological, palaeoecological, and genetic data is available from the literature (Buiteveld et al. 2007; Magri 2008). All these data provide a good start to evaluate the genetic diversity of beech plots established within CLIMO along the distribution range of the species.

Twenty to twenty-five European beech trees were sampled from 12 plots of study 2, including nine countries (Bosnia, Bulgaria, Romania, Hungary, Slovakia Poland, Germany, Italy, and Spain). Six highly variable nuclear microsatellite markers revealed an equilibrated distribution of the genetic variation along the CLIMO plots. As expected, the genetic distance increased with the geographic distance among the study plots, and the highest variation was detected within several beech plots of the western Balkan Peninsula. This region is considered to be an important glacial refuge and a genetic hotspot for many broadleaf species including beech 


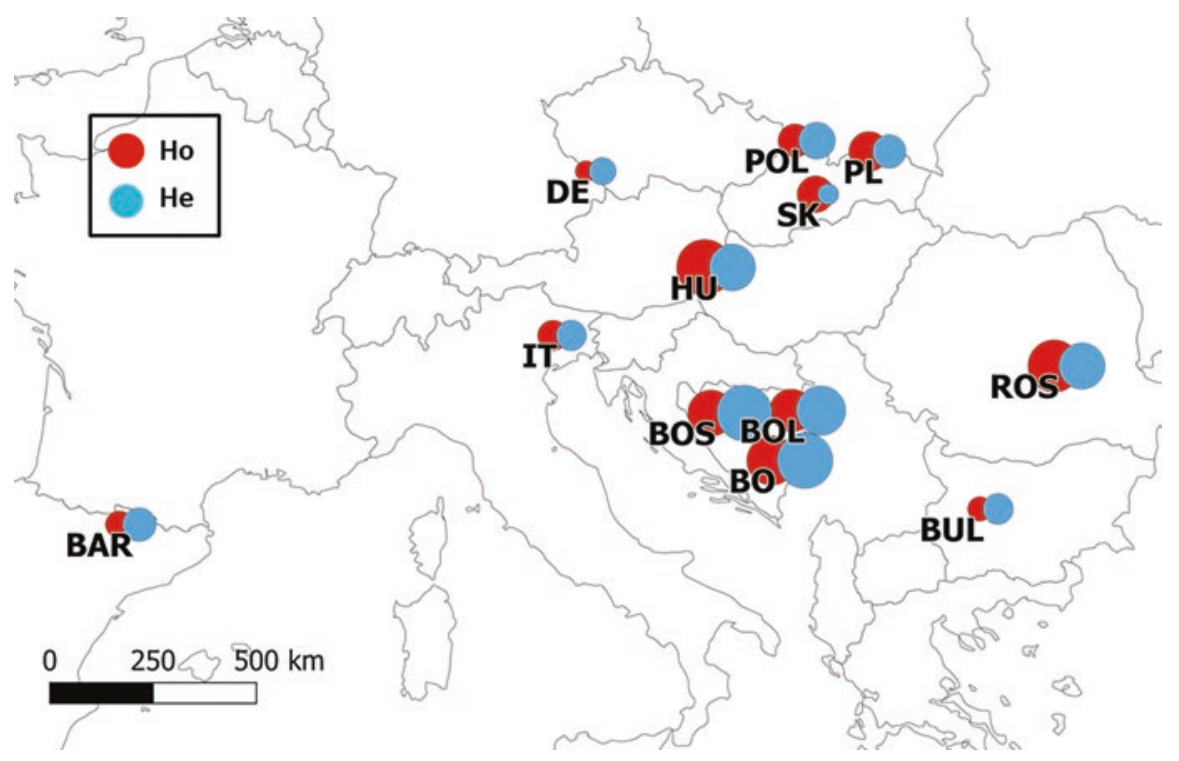

Fig. 5.15 Observed (Ho) and expected heterozygosity (He) of the 12 CLIMO European beech plots. The size of the circle is proportional with the extent of the genetic variation detected in each sample plot. BUL: Bulgaria; BOS, BOL, BO: Bosnia; ROS: Romania; HU: Hungary; SK: Slovakia; POL, PL: Poland; DE: Germany; IT: Italy, BAR: Spain

(Magri et al. 2006). Along with the evaluation of the genetic data in several sampling plots like that from Romania, Slovakia, and Hungary, observed heterozygosity values were higher than the expected heterozygosity values (Fig. 5.15). This means the abundance of heterozygote trees, which might be explained with the sampling strategy, as dominant trees were preferably collected and, most probably, these individuals were in a higher rate heterozygous. Moreover, Petit et al. (2001) reported a pronounced trend of increase in observed heterozygosity with the ageing of the stands. Another explanation should refer to management activity that might have altered the natural genetic composition of the stand. Genetic indices correlated with the climate variables provide further aspects on the frequency distribution of alleles and the environmental variables.

Molecular analysis, detecting neutral genetic variation, is a helpful tool in characterizing the variation of the experimental plots of forest trees and to determine the relationships between groups of individuals or among different plots. However, it should be emphasized that loci with adaptive significance likely were not sampled by these techniques and, accordingly, important adaptive variants of the samples may show different patterns. The selective pressures that affect the populations' adaptiveness are more difficult to be precisely identified, because of the huge genome of forest trees. Mapping of species' entire genome will identify more coding genes and candidates explaining adaptive variation (van Dam 2002). 


\subsection{Trans-Geographic Database of Long-Term Forest Plots in Mountainous Areas}

On the one hand, the establishment of long-term experiments is very laborious, but on the other hand, they provide the unique insight into growth and condition of individual trees and the whole stand. The longer the observation period associated with interdisciplinary data, the better scientific and practical potentials are to study given natural phenomena. Therefore, it is important to make researchers aware of existing long-term plots, report their metadata for judgement whether they are appropriate for a special research question, and get eco-political support for their maintenance over longer time periods. Beyond the CLIMO networks of long-term and temporary plots (Sect. 5.3.4), here we present an exemplary approach on how to make public and available and memorize the existence of a unique set of longterm experiments that answered our research questions, but it may be essential for answering future questions as well.

In the last years, the study of mountain forests has been the focus of increasing research efforts in Europe due to the recognized vulnerability of these systems to the effects of global change (EEA 2012). This has led to the emergence of important research networks, such as the MOUNTFOR initiative (a Project Centre of the European Forest Institute, http://mountfor.fmach.it/) or more recently the SENSFOR and CLIMO Cost Actions (ES1203, CA15226). However, the interest of the European forest science community in mountain forests is not new, as shown by the large number of long-term research plots that have been established across the major mountain ranges of Europe during the last decades. Despite some of the longterm data derived from these plots that have been used in relevant scientific or technical publications (e.g. Martín-Benito et al. 2008; Pretzsch et al. 2015), the existence of the vast majority of these experiments is only known at the local level. Then, its potential use for trans-geographic assessments of forest growth trends (among other issues) is insufficiently exploited. In order to expand the visibility of these longterm experiments and promote future collaborative research initiatives among European scientists, the CLIMO consortium started an action aimed at compiling metadata about existing long-term forest plots established in European mountains. With this purpose, a number of CLIMO representatives conducted different surveys in their respective countries of origin to collect descriptive information on these forest plots, in particular with respect to the following items: the objective, the location (including geographical coordinates and elevation), the year of establishment, the surface, the main tree species and stand age (if even-aged), a short description of the data collected, and the organization and contact details of the person responsible of the plots and related publications (only if available in English). To be included in the surveys, experiments might be established at the latest by the end of the twentieth century and located in mountainous areas. However, these criteria were not applied restrictively (in part because the concept of "mountainous area" varies between a country and another). Accordingly, permanent plots established more recently, but with the aim of evaluating mountain forest responses to climatic changes, were also considered as well as plots located in lowlands under the frame of studies conducted 


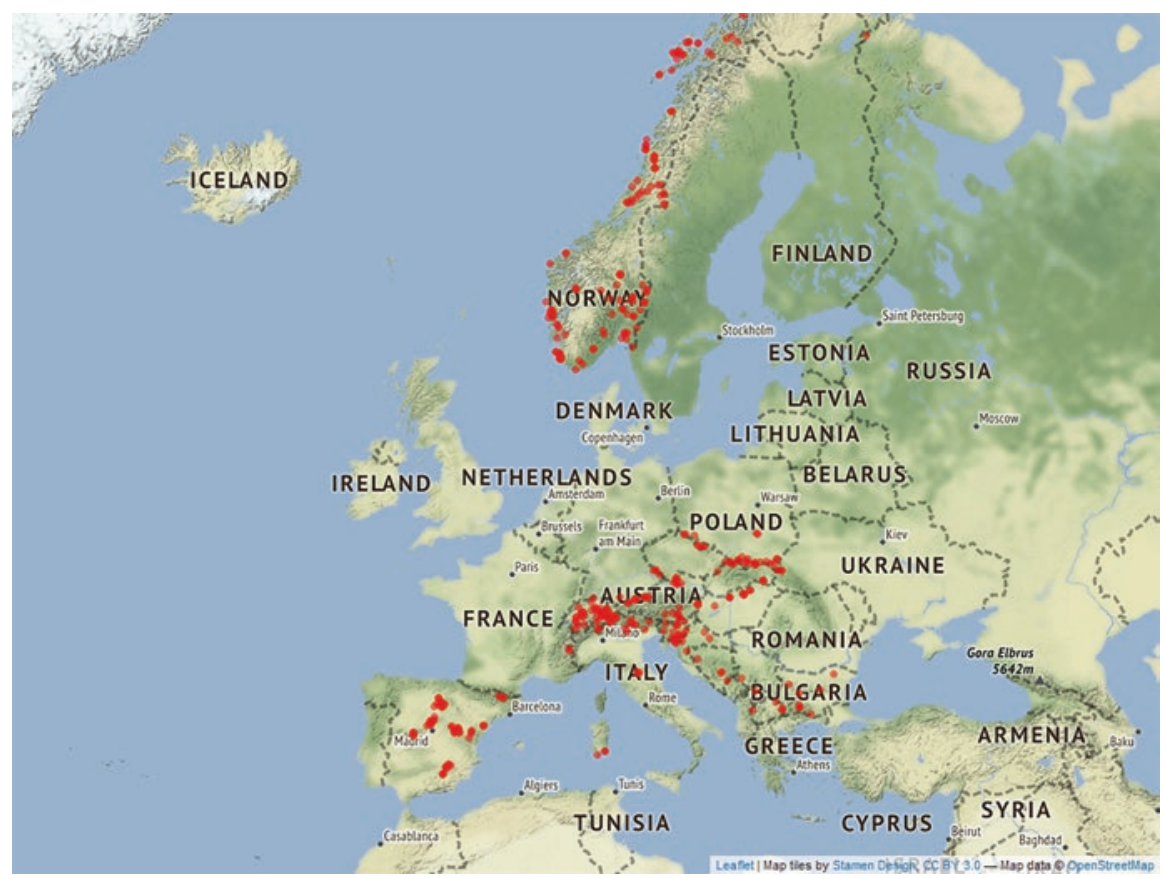

Fig. 5.16 Location of the long-term forest plots included in the ESFONET database

along elevation gradients. Plots established under the frame of large national/ regional forest inventories were not included.

All the collected information was compiled in a common database. In total, the database comprised metadata of 554 permanent plots distributed along 15 countries and covering the major European mountain ranges (Fig. 5.16). A total of 29 species were represented in the plots, with Picea abies, Fagus sylvatica, and Pinus sylvestris being the most dominant (Fig. 5.17). Most of the plots were established during the second half of the twentieth century and about two-third of them were located above 500 meters above sea level (Fig. 5.17). The dataset can be consulted online via a user-friendly interactive application designed with the Shiny $\mathrm{R}$ package (https://climoproject.shinyapps.io/CLIMO_dataset/). The application code is stored in an open repository (GitHub) and allows easy implementation of new data.

Under the frame of the CLIMO Cost Action, this database is considered the basis on which to build a reliable European Smart Forest Network (ESFONET) of longterm experiments strategically distributed across environmental gradients that may provide reliable data to assess the capacity of mountain forests to adapt to climatic changes. The way data is collected and organized determines the level of quality and the accessibility of information, in turn being critical to the success of the network. Implementing systematic procedures for data processing (including data quality evaluation and validation) and testing is, therefore, essential for eliminating errors and reducing uncertainty before data are transferred to a final database (quality assurance, QA) and for controlling the quality of submitted data stored in the final database (quality control, QC). 
A

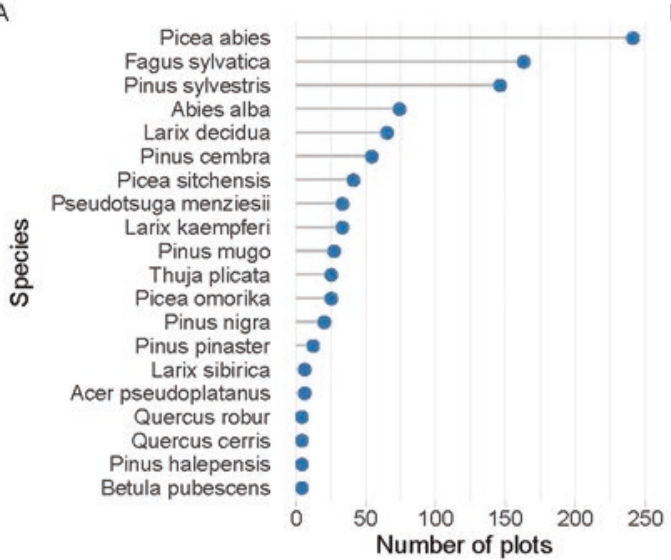

C

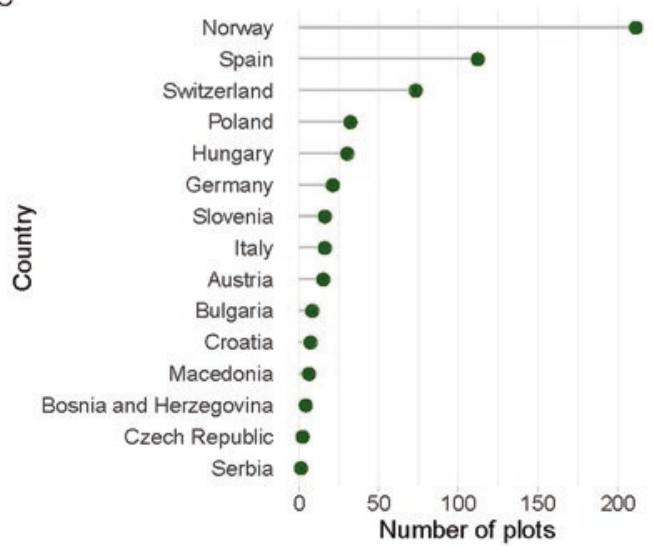

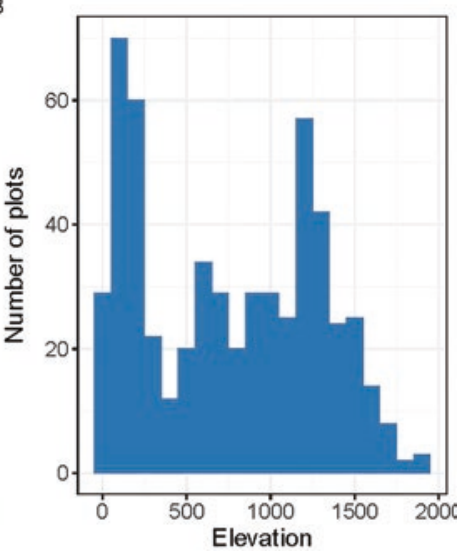

D

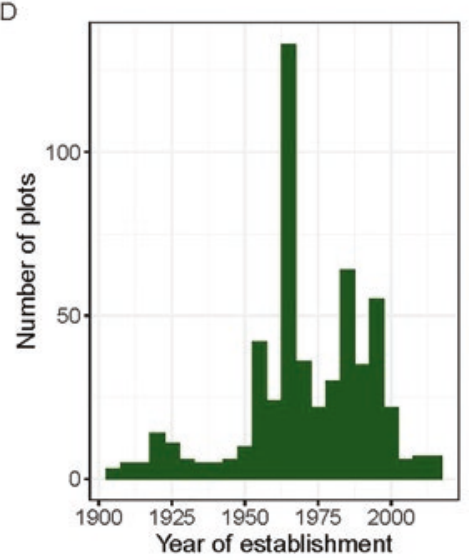

Fig. 5.17 Overview of some of the information collected in the database: (a) the 20 species most present in the database (ordered according to the number of plots in which they are present), (b) distribution of the plots according to the elevation, (c) number of plots located in the participating countries, and (d) distribution of the plots according to the year in which they were established

\subsection{Discussion and Conclusion}

\subsubsection{Exploiting Scattered Long-Term Experiments for Assessing Stand Growth, Resistance, and Climate Smartness by Pooling and Overarching Evaluation of Data}

Despite the credo that substantial information and knowledge require big datasets, overarching pooling and evaluation (Liang et al. 2016; Hilmers et al. 2019; Pretzsch et al. 2019) are still an exception in forest ecology or silviculture. For instance, long-term experiments on thinning were established and treated in a way 
standardized by, e.g. IUFRO (Becking 1953; Johann 1993); however, their evaluation is often restricted to the country or even to the regional level. The tremendous efforts and costs of maintaining long-term experiments may restrain the respective institutions from offering and exchanging their valuable data before having it exploited for their own purposes and publications. However, the evaluations at the state or national level are often of limited value. Samples and evaluations restricted to the state or country level may cover just a small portion of the natural distribution and niche of a tree species. Thus, general and reliable knowledge and relationships between growth and site conditions require trans-geographical evaluations. The broader the range of covered site conditions, the sample size, and the genetic diversity, the more robust and overarching the results for understanding, modelling, and scenario analyses and predictions (Liang et al. 2016; Zeller et al. 2018; Pretzsch et al. 2020a, b).

On the one hand, overarching networks are relevant for monitoring anthropogenic impacts on forest ecosystems and tree species. Local observations may indicate changes, but not their dependency on large-scale environmental conditions, and any shifts in the distribution ranges. On the other hand, overarching networks may contribute to adaptation and mitigation of forest ecosystems to climate change; overarching evaluations and robust growth-site relationships have gained higher importance under climate change. Many studies contribute to better understand the suitability of a given tree species under the expected future climate in, e.g. Central Europe by studying its performance in the north or south, where the species faces already at present the climate predicted for Central Europe in the future (Hanewinkel et al. 2014). This kind of trans-geographical studies is important for detection and remedy of climate changes along latitudinal gradients in the lowlands. And they are even more important along elevation gradients in the mountain forests, where environmental changes may happen in much more narrow space and in shorter time spans with severe consequences for a plenitude of socio-economic forest functions and services (Seidl et al. 2019; Hilmers et al. 2020).

\subsubsection{The Information Potential of Long-Term Versus Inventory Plots}

The pros and cons of long-term experiments compared with temporary plots have been questioned repeatedly (Gadow 1999; Nagel et al. 2012). Often, long-term experiments have been abandoned in order to cut costs. The common reasons for giving up long-term experiments are that forest areas with long-term experiments have to be left out from regular forest operations and that their establishment and survey are costly. In addition, it hardly fits to the contemporary funding organizations and the zeitgeist that it requires a couple of years for getting the first results.

Long-term experiments are also available for other ecosystems. In ecological research (LTER), agriculture and grassland (Rothamsted Research), soil science (LTE), or agroecosystems (LTAE), long-term observations have similar importance 
(Blake 1999; Redman et al. 2004; Körschens 2006) as in forest ecology. Compared with other ecological fields, long-term experiments in forests are even under higher pressure due to their particular longevity and high space consumption. As forest land is often disregarded by politicians and other decision makers and extensively transformed into agricultural areas, urban building, and infrastructures, long-term experiments are frequently sacrificed and valuable long time series of survey discontinued.

It is an often heard, but misleading argument, that forest inventories that have been established during the last few decades at national or enterprise levels can substitute for the information potential of long-term experiments (Gadow 1999). Certainly, temporary plots of forest inventories may be harnessed by innovative Big Data methods, such as geospatial random forests and geostatistical mixed-effects models (e.g. Liang et al. 2016). For obtaining information about the status quo at the enterprise or landscape level, inventories are ideal, as this is what they are designed for. Furthermore, forest inventories provide the data for initializing simulation models, for forest planning and scenario analyses.

In the following, we summarize the main reasons why long-term experiments excel by far the information potential of temporary plots and why they are highly important for ecological monitoring and research (Pretzsch et al. 2019). First, only long-term experiments allow to study the long-term structure and growth development at the tree and stand level. They allow revelation of changing ecological processes, legacy and ecological memory effects, and the long-term consequences of ecosystem alteration. In contrast, temporarily observed inventory plots often lack information about the stand history and any intermediate yield before the plot establishment. This lack of information can be only partly remedied by establishing permanent inventory plots.

Second, long-term experiments are necessary for revelation of the cause-effect relationships between various stand treatment options and the response at the tree and stand level. In contrast to inventory plots, experiments usually analyse tree and stand reaction ceteris paribus, i.e. under parity of tree provenance, stand history, site conditions, and other internal and external factors. Temporary plots may indicate correlations but no evidence for casualties. In contrast to experiments, they may vary in many (even unobserved) traits and not only in the analysed factor of interest (Gamfeldt et al. 2013).

Third, long-term experiments usually cover un-thinned or untreated variants, which, for instance, indicate the maximum stand density, unfertilized conditions, etc. and serve as a reference for the density and growth of other treatments (e.g. thinned, mixed, fertilized plots). In addition, long-term experiments often comprise extreme variants that are usually avoided by forest practice and not covered by inventories. For analysing and modelling forest dynamics, however, extreme treatment variants are often even more informative than standard and business-as-usual variants mainly covered by inventories.

Fourth, only long-term surveys provide a full insight into the growth and yield of the remaining and removal stand, i.e. they quantify the total production since stand 
establishment. This includes also the otherwise not accessible information about the intermediate yield, caused by natural mortality or/and silvicultural treatments.

Fifth, long-term observations often cover a long part of the rotation or even include the subsequent stand generation. In this way, they can reveal effects of changes in environmental conditions at the tree and stand level (Spiecker et al. 1996). As they provide time series of growth and yield data that reach far back in time, they may provide evidence of environmental changes and the human footprint on forest ecosystems. The argument, which similarly long time series can be always obtained by retrospective growth-ring analysis, is only partly true. Retrospective analyses are possible at the individual tree level, but not at all for whole forest stands. This is because only the trees living at the time of sampling can be analysed retrospectively, but not their neighbours and the part of the population, eliminated before due to self-thinning or silvicultural interventions decades before. However, the past development of the population may be important to know its future dynamics (Pretzsch et al. 2021).

\subsubsection{Need for Further Coordination and Standardization of Experimental Design and Set-ups}

The foundation of the International Union of Forest Research Organizations (IUFRO) in 1892 was essential for the coordination and standardization of experimental design and set-ups. The recommendations and definitions for establishment and steering of thinning trials in the early times of IUFRO or its precursor organizations (Verein Deutscher Forstlicher Versuchsanstalten 1873, 1902) had some longterm standardization effects on many kinds of experiments in forests (Becking 1953). However, the objectives and questions of experiments changed and so did the variables and methods to measure. Among others, the measurements were extended to spatial explicit information about coordinates, crown size, stem quality, and vitality at the individual tree level. Natural regeneration, canopy characteristics, as well as variables quantifying the biodiversity, protective functions, recreational, or climate smartness are either added to the variable set of existing experiments. Or they are on the protocol list of new experiments from the beginning on.

Standardizing how to consider mixing proportions would be helpful, as they determine the establishment, steering, and evaluation of experiments (Dirnberger et al. 2017; Halofsky et al. 2018). Standards for height curve, form factor, or diameter growth-diameter functions would harmonize the evaluation of experiments and comparability of their results. Standardization of the result variables of long-term experiments, e.g. regarding merchantable volume, stem volume, stem mass, or total mass, would simplify the common overarching evaluations as realized in some of the studies underlying the book in hand (see Sects. 5.5, 5.6, and 5.7 and Hilmers et al. 2019; Pretzsch et al. 2020a, b; Torresan et al. 2020). 
When establishing new experiments, addressing tree species mixing, natural regeneration, or transitioning from age-class forests to continuous cover forestry requires new standards for quantifying and steering mixing portions, stand density, and canopy cover over regeneration or for the horizontal and vertical stand structuring (del Río et al. 2016).

The date of measurement within the year, the frequency and methodology of measurement, and qualitative assessment of stem quality, crown vitality, etc. need some standardization, necessary for later common evaluation.

The silvicultural prescriptions for steering the experiments need common standards, analogous to the early thinning experiments, but more sophisticated as complex forest requires more detailed protocols for quantitative and reproducible, objective experimental steering (kind, strength, and frequency of interferences).

The common standards for evaluation at the stand and tree level simplify the pooling of data. A report of unique essential results will hopefully advance and support the appreciation and support of long-term experiments due to their unique contribution to forest observation, monitoring, and stewardship.

The standard for data storage and exchange will further support this ambition and save a lot of processing, organization, formatting, and time on the long term.

Trans-geographical projects with their international partner groups have the potential to further update the standard for experiments and observation in forest ecosystems from their establishment to the data storage and common evaluations.

\subsubsection{Maintenance of Both Unmanaged and Managed Observation Plots}

Untreated plots are of special value as reference for natural ecosystem dynamics without direct interference but suitable for revelation of indirect anthropogenic effects. They may reveal and quantify the effects of acid rain (Spiecker et al. 1996), climate change (Pretzsch et al. 2014), and also just local disturbances caused by lowering the groundwater level (Pretzsch and Kölbel 1988) or ozone (Matyssek et al. 2010). The growth and structure in managed forests is often superimposed by management effects in a way that disturbance by management and environmental stress are difficult to separate.

Unmanaged does not mean unmeasured; untreated plots are often accurately measured regarding vitality, growth, mortality, standing, and lying deadwood. Already the early thinning experiments provided un-thinned plots as reference; the inventory of all dropout trees provided information about the total yield over the whole rotation, not available without such concepts.

The reference plots offer information about maximum stand density, selfthinning, natural intra- and interspecific competition processes, and disturbances. They provide information for the derivation of basic relationships (Assmann 2013), model parameterization (Pretzsch et al. 2002), and development of silvicultural prescriptions for both monospecific and especially mixed-species stands (Kelty 1992; 
Dieler et al. 2017). Any treatment experiments gain in value if untreated plots are nearby and demonstrate the impact of treatment by visual and quantitative comparison of both. In this network, we used the untreated plots for exploring trends in stand and tree growth that might be superimposed by various kinds of management effects on specifically treated experimental plots or un-specifically managed inventory plots (von Gadow and Kotze 2014). Plots with trees growing solitarily would be of similar interest but are even rarer (Kuehne et al. 2013; Uhl et al. 2015). They would reflect the vitality, growth, allometry, and ageing without competition, which would be of similar interest (for understanding, model parameterization, biomonitoring) as growth under self-thinning and without treatment.

\subsubsection{The Relevance and Perspectives of Common Platforms for Forest Research}

The use of both long-term experiments as well as temporary plots with increment coring for detecting growth trends reveals the shortcomings of temporal plots for reliable information of growth trends. There are shortcomings of using temporary plots for tree-level evaluations as well as using them for retrospective stand-level growth trend diagnosis.

Trend statements about tree-level growth based on increment cores from temporary plots may be misleading if the tree history is unknown. Sampling in mature stands may lead to biased results, as it means a sampling of survivors that may not represent the mean growth of the population. Notice that a European beech stand may start with a million trees or so and arrive at 50-100 trees per hectare in the mature phase with a mortality rate of $1-7 \%$. The dropout trees may be less vital and lower in growth, and the growth of the remaining trees is higher than the mean (Nehrbass-Ahles et al. 2014). The effects of unknown silvicultural treatment in the past, natural suppression in the understorey, or biotic and abiotic damages (e.g. browsing, acid rain) may have influenced the life history and still have a memory effects of the present and future growth of the trees. By selecting sample trees with long and large crowns indicating permanent dominance and with tree-ring patterns without narrow ring phases by suppression may reduce such biases but cannot completely avoid them.

By sampling on long-term experiments or observation plots, in contrast, it is possible to select representative trees without prior charge by suppression, silvicultural impact, or damages that may interfere with the normally sigmoid growth-age relation.

For the detection of any stand growth trend, information about the growth of trees of various social positions in the stand and about tree removal, mortality, and stand structure in the past is even more important (Torresan et al. 2020). In this platform, we tried to avoid misjudgements by selection of stands un-thinned at least in the near past, by sampling trees for retrospective growth calculation from all biosocial classes over the whole stem diameter range and by assessing tree mortality 
via stump inventory. In this way, stand growth can be derived retrospectively (Heym et al. 2017, 2018).

All these shortcomings underline the advantages of maintaining a network of long-term experiments for monitoring the vitality and growth of forests (Pretzsch et al. 2019) that after all cover one third of the land surface in Europe and deserve special stewardship, especially in the mountain areas with their manifold ecological, economical, and socio-economic functions and services (Biber et al. 2015; Dieler et al. 2017).

Acknowledgements The authors would like to acknowledge networking support by the COST (European Cooperation in Science and Technology) Action CLIMO (Climate-Smart Forestry in Mountain Regions - CA15226) financially supported by the EU Framework Programme for Research and Innovation HORIZON 2020. This publication is part of a project that has received funding from the European Union's HORIZON 2020 research and innovation programme under the Marie Skłodowska-Curie grant agreement No 778322. Acknowledgements are also due to the European Union for funding the project "Mixed species forest management. Lowering risk, increasing resilience (REFORM)" (\# 2816ERA02S under the framework of Sumforest ERA-Net). Further, we would like to thank the Bayerische Staatsforsten (BaySF) for providing the observational plots and to the Bavarian State Ministry of Food, Agriculture, and Forestry for the permanent support of the project W 07 "Long-term experimental plots for forest growth and yield research" (\#7831-26625-2017). We also thank the Forest Research Institute, ERTI Sárvár, Hungary, for assistance and for providing observational plots. Research at Polish case studies was additionally supported by the Polish Government MNiSW 2018-2021 matching fund (W117/H2020/2018). In Slovenia, the study was additionally supported by the research programme P4-0059 Forest, forestry, and renewable forest resources. In Bosnia and Hercegovina, collecting data from plots is supported by the Ministry of Civil Affairs of Bosnia and Herzegovina.

\section{References}

Anderson-Teixeira KJ, Davies SJ, Bennett AC et al (2015) CTFS-Forest GEO: a worldwide network monitoring forests in an era of global change. Glob Chang Biol 21(2):528-549

Assmann E (2013) The principles of forest yield study: studies in the organic production, structure, increment and yield of forest stands. Elsevier

Bauhus J, Forrester DI, Pretzsch H (2017) From observations to evidence about effects of mixedspecies stands. In: Pretzsch H, Forrester DI, Bauhus J (eds) Mixed-species forests. Springer, Berlin, Heidelberg, pp 27-71

Becker A, Bugmann H (2001) Global change and mountains regions - an IGBP initiative for collaborative research. In: Visconti G, Beniston M, Iannorelli ED, Diego B (eds) Global change and protected areas. Kluwer Academic, Dordrecht, pp 3-10

Becking JH (1953) Einige Gesichtspunkte für die Durchführung von vergleichenden Durchforstungsversuchen in gleichaltrigen Beständen. Proceedings of the $11^{\text {th }}$ IUFRO Congress 1953, Rome, pp 580-582

Biber P, Borges JG, Moshammer R et al (2015) How sensitive are ecosystem services in European forest landscapes to silvicultural treatment? Forests 6(5):1666-1695

Blake J (1999) Overcoming the 'value-action gap' in environmental policy: tensions between national policy and local experience. Local Environ 4(3):257-278

Bowditch E, Santopuoli G, Binder F et al (2020) What is Climate-Smart Forestry? A definition from a multinational collaborative process focused on mountain regions of Europe. Ecosyst Serv 43:101113. https://doi.org/10.1016/j.ecoser.2020.101113 
Buiteveld J, Vendramin GG, Leonardi S et al (2007) Genetic diversity and differentiation in European beech (Fagus sylvatica L.) stands varying in management history. For Ecol Manag 247:98-106

Carter MR, Gregorich EG (2007) Soil sampling and methods of analysis. CRC press

Cherubini P, Dobbertin M, Innes JL (1998) Potential sampling bias in long-term forest growth trends reconstructed from tree rings: a case study from the Italian Alps. For Ecol Manag 109(1-3):103-118

de Martonne E (1926) Une nouvelle fanction climatologique. L'indice d'aridité. La Meteriologie. Gauthier-Villars, Paris, pp 449-458

del Rio M, Condés S, Pretzsch H (2014) Analyzing size-symmetric vs. size-asymmetric and intravs. inter-specific competition in beech (Fagus sylvatica L.) mixed stands. For Ecol Manag 325:90-98. https://doi.org/10.1016/j.foreco.2014.03.047

del Río M, Pretzsch H, Alberdi I et al (2016) Characterization of the structure, dynamics, and productivity of mixed-species stands: review and perspectives. Eur J For Res 135(1):23-49

del Río M, Vergarechea M, Hilmers T et al (2021) Effects of elevation-dependent climate warming on intra- and inter-specific growth synchrony in mixed mountain forests. For Ecol Manag 479. https://doi.org/10.1016/j.foreco.2020.118587

Dieler J, Uhl E, Biber P et al (2017) Effect of forest stand management on species composition, structural diversity, and productivity in the temperate zone of Europe. Eur J For Res 136(4):739-766

Dirnberger G, Sterba H, Condés S et al (2017) Species proportions by area in mixtures of Scots pine (Pinus sylvestris L.) and European beech (Fagus sylvatica L.). Eur J For Res 136(1):171-183

Dorado-Liñán I, Valbuena-Carabaña M, Cañellas I et al (2020) Climate change synchronizes growth and iWUE across species in a temperate-submediterranean mixed oak forest. Front Plant Sci 11:706

EEA (2012) Climate change, impacts and vulnerability in Europe 2012. An indicator-based report, EEA report no 12/2012, European Environment Agency, Copenhagen

Ettinger AK, Ford KR, HilleRisLambers J (2011) Climate determines upper, but not lower, altitudinal range limits of Pacific Northwest conifers. Ecology 92(6):1323-1331

Falk DA, Richards CM, Arlee M et al (2006) Population and ecological genetics. In: Falk DA, Palmer MA, Zedler JB (eds) Restoration ecology. Foundations of restoration ecology. Island Press, Washington, DC, pp 14-41

Ferretti M, Fischer R (2013) Forest monitoring: methods for terrestrial investigations in Europe with an overview of North America and Asia. Vol. 12 of Developments in environmental science. Elsevier, Amsterdam

Forest Europe (2015) Forest Europe C\&I set for sustainable forest management (SFM) (https:// foresteurope.org/sfm-criteria-indicators/)

Forrester DI, Tachauer IH, Annighoefer P et al (2017) Generalized biomass and leaf area allometric equations for European tree species incorporating stand structure, tree age and climate. For Ecol Manag 396:160-175

Franklin JF (1989) Importance and justification of long-term studies in ecology. In: Likens GE (ed) Long-term studies in ecology. Springer, New York, pp 3-19

Gadow von K (1999) Datengewinnung für Baumhöhenmodelle - permanente und temporäre Versuchsflächen, Intervallflächen. Centralblatt für das gesamte Forstwesen 116(1/2):81-90

Gamfeldt L, Snäll T, Bagchi R et al (2013) Higher levels of multiple ecosystem services are found in forests with more tree species. Nat Commun 4:1340

Gazol A, Camarero JC (2016) Functional diversity enhances silver fir growth resilience to an extreme drought. J Ecol 104(4):1063-1075

Grissino-Mayer HD, Fritts HC (1997) The International Tree-Ring Data Bank: an enhanced global database serving the global scientific community. The Holocene 7(2):235-238

Halofsky JE, Andrews-Key SA, Edwards JE et al (2018) Adapting forest management to climate change: the state of science and applications in Canada and the United States. For Ecol Manag 421:84-97

Hanewinkel M, Kuhn T, Bugmann $\mathrm{H}$ et al (2014) Vulnerability of uneven-aged forests to storm damage. Forestry 87(4):525-534 
Harmon ME, Pabst RJ (2015) Testing predictions of forest succession using long-term measurements: 100 years of observation in the Oregon Cascades. J Veg Sci 26:722-732

Harris I, Osborn TJ, Jones P et al (2020) Version 4 of the CRU TS monthly high-resolution gridded multivariate climate dataset. Sci Data 7(1):1-18. https://doi.org/10.1038/s41597-020-0453-3

Hernández L, Camarero JJ, Gil-Peregrín E et al (2019) Biotic factors and increasing aridity shape the altitudinal shifts of marginal Pyrenean silver fir populations in Europe. For Ecol Manag 432:558-567. https://doi.org/10.1016/j.foreco.2018.09.037

Heym M, Ruíz-Peinado R, del Río M et al (2017) EuMIXFOR empirical forest mensuration and ring width data from pure and mixed stands of Scots pine (Pinus sylvestris L.) and European beech (Fagus sylvatica L.) through Europe. Ann For Sci 74(3):63

Heym M, Bielak K, Wellhausen K et al (2018) A new method to reconstruct recent tree and stand attributes of temporary research plots: new opportunity to analyse mixed forest stands. In: Gonçalves AC (ed) Conifers. IntechOpen, Rijeka, pp 25-45

Hilmers T, Avdagić A, Bartkowicz L et al (2019) The productivity of mixed mountain forests comprised of Fagus sylvatica, Picea abies, and Abies alba across Europe. Forestry (Lond) 92(5):512-522. https://doi.org/10.1093/forestry/cpz035

Hilmers T, Biber P, Knoke T et al (2020) Assessing transformation scenarios from pure Norway spruce to mixed uneven-aged forests in mountain areas. Eur J For Res. https://doi.org/10.1007/ s10342-020-01270-y

Jiang Z, Liu H, Wang H et al (2020) Bedrock geochemistry influences vegetation growth by regulating the regolith water holding capacity. Nat Commun 11:2392. https://doi.org/10.1038/ s41467-020-16156-1

Johann K (1993) DESER-Norm 1993. Normen der Sektion Ertragskunde im Deutschen Verband Forstlicher Forschungsanstalten zur Aufbereitung von waldwachstumskundlichen Dauerversuchen. Proc Dt Verb Forstl Forschungsanst, Sek Ertragskd, in UnterreichenbachKapfenhardt, pp 96-104

Kangas A, Maltamo M (2006) Forest inventory: methodology and applications. Springer, Dordrecht, London

Kapos V, Rhind J, Edwards M, Price MF, Ravilious C (2000) Developing a map of the world's mountain forests. Forests in sustainable mountain development: a state of knowledge report for 2000. Task Force on Forests in Sustainable Mountain Development, pp 4-19

Kelty MJ (1992) Comparative productivity of monocultures and mixed-species stands. In: The ecology and silviculture of mixed-species forests. Springer, Dordrecht, pp 125-141

Körschens M (2006) The importance of long-term field experiments for soil science and environmental research - a review. Plant Soil Environ 52(Special Issue):1-8

Körner C, Paulsen J, Spehn EM (2011) A definition of mountains and their bioclimatic belts for global comparisons of biodiversity data. Alpine Botany, 121(2):73-78

Kramer K, Degen B, Buschbom J et al (2010) Modelling exploration of the future of European beech (Fagus sylvatica L.) under climate change - range, abundance, genetic diversity and adaptive response. For Ecol Manag 259:2213-2222

Kuehne C, Kublin E, Pyttel P et al (2013) Growth and form of Quercus robur and Fraxinus excelsior respond distinctly different to initial growing space: results from 24-year-old Nelder experiments. J For Res 24(1):1-14

Liang J, Crowther TW, Picard N (2016) Positive biodiversity-productivity relationship predominant in global forests. Science 354(6309):aaf8957. https://doi.org/10.1126/science.aaf8957

Lloret F, Keeling EG, Sala A (2011) Components of tree resilience: effects of successive lowgrowth episodes in old ponderosa pine forests. Oikos 120(12):1909-1920

Lutz JA (2015) The evolution of long-term data for forestry: large temperate research plots in an era of global change. Northw Sci 89:255-269

Magri D (2008) Patterns of post-glacial spread and the extent of glacial refugia of European beech (Fagus sylvatica). J Biogeogr 35:450-463 
Magri D, Vendramin GG, Comps B et al (2006) A new scenario for the Quaternary history of European beech populations: palaeobotanical evidence and genetic consequences. New Phytol 171:199-221

Martín-Benito D, Gea-Izquierdo G, del Río M et al (2008) Long-term trends in dominant-height growth of black pine using dynamic models. For Ecol Manag 256:1230-1238

Matyssek R, Wieser G, Ceulemans R et al (2010) Enhanced ozone strongly reduces carbon sink strength of adult beech (Fagus sylvatica) - resume from the free-air fumigation study at Kranzberg Forest. Environ Pollut 158(8):2527-2532

Nagel J, Spellmann H, Pretzsch H (2012) Zum Informationspotenzial langfristiger forstlicher Versuchsflächen und periodischer Waldinventuren für die waldwachstumskundliche Forschung. Allg For Jagdzeitung 183(5/6):111-116

Nehrbass-Ahles C, Babst F, Klesse S et al (2014) The influence of sampling design on tree-ringbased quantification of forest growth. Glob Chang Biol 20(9):2867-2885

Nogués-Bravo D, Araújoc MB, Erread MP et al (2007) Exposure of global mountain systems to climate warming during the 21st century. Glob Environ Chang 17:420-428

Palmer WC (1965) Meteorological drought, U.S. Weather Bureau, research paper 45. U.S. Department of Commerce, Weather Bureau, Washington, DC

Pennock DJ, van Kessel C (1997) Clear-cut forest harvest impacts on soil quality indicators in the mixedwood forest of Saskatchewan, Canada. Geoderma 75:13-32

Petit RJ, Bialozyt R, Brewer S et al (2001) From spatial patterns of genetic diversity to postglacial migration processes in forest trees. In: Silvertown J, Antonovics J (eds) Integrating ecology and evolution in a spatial context. Blackwell Science, Oxford, pp 295-318

Pfatrisch M (2019) Assessment of climate smartness. Master thesis. Technical University of Munich, Freising, 115p

Porth I, El-Kassaby YA (2014) Assessment of the genetic diversity in forest tree populations using molecular markers. Diversity 6:283-295

Pretzsch H (2009) Forest dynamics, growth and yield. Springer, Berlin, $664 \mathrm{p}$

Pretzsch H, Biber P (2016) Tree species mixing can increase maximum stand density. Can J For Res 46:1179-1193. https://doi.org/10.1139/cjfr-2015-0413

Pretzsch H, Kölbel M (1988) Einfluß von Grundwasserabsenkungen auf das Wuchsverhalten der Kiefernbestände im Gebiet des Nürnberger Hafens - Ergebnisse ertragskundlicher Untersuchungen auf der Weiserflächenreihe Nürnberg 317. Forstarchiv 59(3):89-96

Pretzsch H, Biber P, Durský J (2002) The single tree-based stand simulator SILVA: construction, application and evaluation. For Ecol Manag 162(1):3-21

Pretzsch H, Biber P, Schütze G et al (2014) Forest stand growth dynamics in Central Europe have accelerated since 1870. Nat Commun 5:4967. https://doi.org/10.1038/ncomms5967

Pretzsch H, Biber P, Uhl E et al (2015) Long-term stand dynamics of managed spruce-fir-beech mountain forests in Central Europe: structure, productivity and regeneration success. Forestry 88(4):407-428. https://doi.org/10.1093/forestry/cpv013

Pretzsch H, Schütze G, Biber P (2018) Drought can favour the growth of small in relation to tall trees in mature stands of Norway spruce and European beech. For Ecosyst 5(1):20

Pretzsch H, del Rio M, Biber P et al (2019) Maintenance of long-term experiments for unique insights into forest growth dynamics and trends: review and perspectives. Eur J For Res 138(1):165-185. https://doi.org/10.1007/s10342-018-1151-y

Pretzsch H, Hilmers T, Biber P et al (2020a) Evidence of elevation-specific growth changes of spruce, fir, and beech in European mixed-mountain forests during the last three centuries. Can J For Res 50(7):689-703. https://doi.org/10.1139/cjfr-2019-0368

Pretzsch H, Hilmers T, Uhl E et al (2020b) European beech stem diameter grows better in mixed than in mono-specific stands at the edge of its distribution in mountain forests. Eur J For Res. https://doi.org/10.1007/s10342-020-01319-y

Pretzsch H, del Río M, Giammarchi F et al (2021) Changes of tree and stand growth. Review and implications. IIn: Managing Forest Ecosystems, Vol. 40, Tognetti R, Smith M, Panzacchi P (Eds): Climate-Smart Forestry in Mountain Regions. Springer Nature, Switzerland, AG 
Raison RJ, Khanna PK (2011) Possible impacts of climate change on forest soil health, Chapter 12. In: Singh BP et al (eds) Soil health and climate change, Soil biology 29. Springer, Berlin, Heidelberg. https://doi.org/10.1007/978-3-642-20256-8_12

Redman CL, Grove JM, Kuby LH (2004) Integrating social science into the long-term ecological research (LTER) network: social dimensions of ecological change and ecological dimensions of social change. Ecosystems 7(2):161-171

Ruiz-Benito P, Vacchiano G, Lines ER et al (2020) Available and missing data to model impacts of climate change on European forests. Ecol Model 416:108870

Sagarin R, Pauchard A (2010) Observational approaches in ecology open new ground in a changing world. Front Ecol Environ 8(7):379-386

Santopuoli G, Temperli C, Alberdi I et al (2020) Pan-European sustainable forest management indicators for assessing Climate-Smart Forestry in Europe. Can J For Res. https://doi. org/10.1139/cjfr-2020-0166

Seidl R, Albrich K, Erb K et al (2019) What drives the future supply of regulating ecosystem services in a mountain forest landscape? For Ecol Manag 445:37-47. https://doi.org/10.1016/j. foreco.2019.03.047

Spiecker H, Mielikäinen K, Köhl M, Skovsgaard JP (eds) (1996) Growth trends in European forests, Europ For Inst, Res Rep, vol 5. Springer, Heidelberg, 372 p

Tognetti R, Scarascia Mugnozza G, Hofer T (eds) (2017) Mountain watersheds and ecosystem services: balancing multiple demands of forest management in head-watersheds, EFI technical report 101. EFI, Joensuu, $191 \mathrm{p}$

Tognetti R, Valentini R, Belelli Marchesini L, Gianelle D, Panzacchi P, Marshall JD (2021) Continuous monitoring of tree responses to climate change for smart forestry - a cybernetic web of trees. In: Managing Forest Ecosystems, Vol. 40, Tognetti R, Smith M, Panzacchi P (Eds): Climate-Smart Forestry in Mountain Regions. Springer Nature, Switzerland, AG

Tomppo E, Gschwantner T, Lawrence M et al (2010) National forest inventories. Pathways for common reporting. Springer, Dordrecht

Torresan C, del Río M, Hilmers T et al (2020) Importance of tree species size dominance and heterogeneity on the productivity of spruce-fir-beech mountain forest stands in Europe. For Ecol Manag 457:117716

Torresan C, Luyssaert S, Filippa G, Imangholiloo M, Gaulton R (2021) Remote sensing technologies for assessing climate-smart criteria in mountain forests. In: Managing Forest Ecosystems, Vol. 40, Tognetti R, Smith M, Panzacchi P (Eds): Climate-Smart Forestry in Mountain Regions. Springer Nature, Switzerland, AG

Uhl E, Biber P, Ulbricht M et al (2015) Analysing the effect of stand density and site conditions on structure and growth of oak species using Nelder trials along an environmental gradient: experimental design, evaluation methods, and results. For Ecosyst 2(1):17

van Dam BC (2002) EUROPOP: genetic diversity in river populations of European black poplar for evaluation of biodiversity, conservation strategies, nature development and genetic improvement. In: Dam BC, Bordács S (eds) Genetic diversity in river populations of European black poplar. Proceedings of International Symposium Szekszárd, 16-20 May 2001. Csiszár nyomda, Budapest, pp 15-31

van der Maaten-Theunissen M, van der Maaten E, Bouriaud O (2015) PointRes: An R package to analyze pointer years and components of resilience. Dendrochronologia 35:34-38

van Mantgem PJ, Stephenson NL (2007) Apparent climatically induced increase of tree mortality rates in a temperate forest. Ecol Lett 10:909-916

Verein Deutscher Forstlicher Versuchsanstalten (1873) Anleitung für Durchforstungsversuche. In: Ganghofer von A ed. (1884) Das Forstliche Versuchswesen. Schmid'sche Buchhandlung, Augsburg, vol 2, pp. 247-253

Verein Deutscher Forstlicher Versuchsanstalten (1902) Beratungen der vom Vereine Deutscher Forstlicher Versuchsanstalten eingesetzten Kommission zur Feststellung des neuen Arbeitsplanes für Durchforstungs- und Lichtungsversuche. AFJZ 78:180-184 
Versace S, Gianelle D, Frizzera L et al (2019) Prediction of competition indices in a Norway Spruce and Silver Fir-Dominated Forest Using Lidar Data. Remote Sens 11(23):2734

Versace S, Gianelle D, Garfi V et al (2020) Interannual radial growth sensitivity to climatic variations and extreme events in mixed-species and pure forest stands of silver fir and European beech in the Italian Peninsula. Eur J For Res:1-19

Vicente-Serrano SM, Beguería S, López-Moreno JI (2010) A multiscalar drought index sensitive to global warming: the standardized precipitation evapotranspiration index. J Clim 23:1696-1718

von Gadow K, Kotze H (2014) Tree survival and maximum density of planted forests - observations from South African spacing studies. For Ecosyst 1(1):21

Wohner C, Peterseil J, Poursanidis D et al (2019) DEIMS-SDR - a web portal to document research sites and their associated data. Eco Inform 51:15-24

Zeller L, Liang J, Pretzsch H (2018) Tree species richness enhances stand productivity while stand structure can have opposite effects, based on forest inventory data from Germany and the United States of America. For Ecosyst 5(1):4

Open Access This chapter is licensed under the terms of the Creative Commons Attribution 4.0 International License (http://creativecommons.org/licenses/by/4.0/), which permits use, sharing, adaptation, distribution and reproduction in any medium or format, as long as you give appropriate credit to the original author(s) and the source, provide a link to the Creative Commons license and indicate if changes were made.

The images or other third party material in this chapter are included in the chapter's Creative Commons license, unless indicated otherwise in a credit line to the material. If material is not included in the chapter's Creative Commons license and your intended use is not permitted by statutory regulation or exceeds the permitted use, you will need to obtain permission directly from the copyright holder. 